Canadian

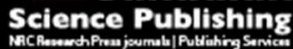

Canadian Geotechnical Journal Revue canadienne de géotechnique

\title{
Impact Mechanisms of Granular and Viscous Flows on Rigid and Flexible Barriers
}

\begin{tabular}{|r|l|}
\hline Journal: & Canadian Geotechnical Journal \\
\hline Manuscript ID & cgj-2016-0128.R1 \\
\hline Manuscript Type: & Article \\
\hline Date Submitted by the Author: & $20-$ Jul-2016 \\
\hline Complete List of Authors: & $\begin{array}{l}\text { Ng, C.W.W.; Hong Kong University of Science and Technology, Department } \\
\text { of Civil and Environmental Engineering } \\
\text { Song, Dongri; The Hong Kong University of Science and Technology, } \\
\text { Department of Civil and Environmental Engineering } \\
\text { Choi, Clarence; Hong Kong University of Science and Technology, } \\
\text { Department of Civil and Environmental Engineering } \\
\text { Liu, L. H. D.; Hong Kong University of Science and Technology, } \\
\text { Department of Civil and Environmental Engineering } \\
\text { Kwan, J.S.H.; 12/F Civil Engineering and Development Department } \\
\text { Building, } \\
\text { Koo, R.C.H.; Geotechnical Engineering Office, } \\
\text { Pun, W.K.; Geotechnical Engineering Office }\end{array}$ \\
\hline Keyword: & \begin{tabular}{l} 
centrifuge modelling, impact, granular flow, viscous flow, flexible barrier \\
\hline
\end{tabular} \\
\hline
\end{tabular}




\section{General information of the article}

Type of paper: Article

Title: Impact Mechanisms of Granular and Viscous Flows on Rigid and Flexible Barriers

Authors: C. W. W. Ng, D. Song*, C. E. Choi, L. H. D. Liu, J. S. H. Kwan, R. C. H. Koo, and W. K. Pun

*Corresponding author

\section{Information of the authors}

Corresponding author: Mr. D Song

Research student, Department of Civil and Environmental Engineering, Hong Kong

University of Science and Technology, Clear Water Bay, Kowloon, Hong Kong.

E-mail: dsong@connect.ust.hk

Co-author: Dr C. W. W. Ng

Chair Professor, Department of Civil and Environmental Engineering, Hong Kong

University of Science and Technology, Clear Water Bay, Kowloon, Hong Kong.

E-mail: cecwwng@ust.hk

Co-author: Dr C. E. Choi

Research Assistant Professor, Department of Civil and Environmental Engineering, Hong Kong University of Science and Technology, Clear Water Bay, Kowloon, Hong Kong.

E-mail:ceclarence@,ust.hk

Co-author: Mr L. H. D. Liu

Research student, Department of Civil and Environmental Engineering, Hong Kong University of Science and Technology, Clear Water Bay, Kowloon, Hong Kong. E-mail: hliuaq@connect.ust.hk

Co-author: Dr J. S.H. Kwan

Senior Engineer, Geotechnical Engineering Office, Civil Engineering and Development Department, HKSAR

E-mail: juliankwan@cedd.gov.hk

Co-author: Mr R. C. H. Koo

Engineer, Geotechnical Engineering Office, Civil Engineering and Development 
Department, HKSAR

E-mail: raymondchkoo@cedd.gov.hk

Co-author: Mr W. K. Pun

Deputy Head, Geotechnical Engineering Office, Civil Engineering and Development Department, HKSAR

E-mail: wkpun@cedd.gov.hk

\section{Impact Mechanisms of Granular and Viscous Flows on Rigid and Flexible Barriers}

C. W. W. Ng, D. Song, C. E. Choi, L. H. D. Liu, J. S. H. Kwan, R. C. H. Koo, and W. K. Pun

Abstract: Structural countermeasures such as rigid and flexible barriers are commonly installed in mountainous regions to intercept mass-wasting processes. Without sufficient and reliable comparable physical data, the study of impact mechanisms remains difficult and not well understood. In this study, a newly developed flexible model barrier together with a rigid barrier are used to simulate either dry granular or viscous liquid impacts on these model barriers in a geotechnical centrifuge. The novel flexible barrier is made of four instrumented cables controlled by spring mechanisms to replicate a bilinear prototype loading response. Tests revealed that regardless of barrier type, both dry granular and viscous flows could have similar frontal dynamic impact coefficients around unity. Compared with the kinetic energy of flow mass $(\sim 10 \mathrm{MJ})$, only $249 \mathrm{~kJ}$ of flexible barrier energy capacity was mobilized. This implies that debris-resisting barriers may only be required to intercept the dynamic flow front as the subsequent flow energy may mainly be dissipated through internal shearing. Attributing to the large deformation of the flexible barrier, the granular static load acting on the flexible barrier could be $39 \%$ lower than that on the rigid barrier, resulting in an active failure mode and a lower earth pressure. 
Keywords: impact; granular flow; viscous flow; flexible barrier; debris-barrier interaction; centrifuge modelling

\section{Introduction}

Mass-wasting processes, such as debris flows and avalanches, can occur without warning and carry large momentum as they surge downslope (Iverson 1997; Hungr et al. 2014). Conventionally, to intercept these hazardous phenomena, rigid reinforced concrete barriers are installed along the predicted flow path. However, over the past decade, rockfall flexible barriers have been hit by landslides and have proven to be quite effective (Duffy and DeNatale 1996; Wendeler et al. 2007; Kwan et al. 2014). A typical flexible barrier comprises a net, energy dissipating elements, posts and cables. The energy absorption characteristic of a flexible barrier relies principally on its energy dissipating devices (Wendeler et al. 2008). Flexible barriers can more easily blend in with its surroundings rendering it a more environmentally-friendly option compared to large reinforced concrete barriers. As flexible barriers emerge as the structural countermeasure of choice to impede landslides, there is certainly a need to better understand its impact mechanism.

The impact force $(F)$ on barriers is usually predicted using the hydrodynamic approach (Hübl et al. 2013) which is given as follows

$F=\alpha \rho v^{2} h w$

or alternatively, the hydrostatic approach, which adopts the form as follows

$F=0.5 \kappa \rho g h^{2} w$

where $\alpha$ is dynamic pressure coefficient, $\kappa$ is static pressure coefficient, $\rho$ is the density of the 
flow $\left(\mathrm{kg} / \mathrm{m}^{3}\right), v$ is frontal velocity $(\mathrm{m} / \mathrm{s})$, and $h, w$ are the flow depth and barrier width $(\mathrm{m})$, respectively. The dynamic/static pressure coefficients are semi-empirical in nature. In practice, it is common to assume that the entire flow mass impacts the barrier without load attenuation (Koo et al. 2016). It is clear that further research is required to bear additional insight and physical data to help understand the dynamic loading induced on both rigid and flexible barriers.

There exist several scientific approaches for researchers to investigate and obtain physical test data for landslide flow impact on structural countermeasures. These methodologies include full-scale instrumented tests, small-scale flume, and centrifuge modelling. Given the poor temporal predictability of natural mass-wasting events and the high costs associated with large-scale testing, little well documented and properly instrumented prototype data is readily available in the literature. The Illgraben torrent in the Illbach River is a relatively active torrent that allows researchers to study natural debris flow dynamics (McArdell et al. 2007). In addition, a series of check dams and a flexible barrier is installed within the Illbach River. Impact on an instrumented flexible barrier was reported by Wendeler et al. (2007). The flexible barrier was instrumented with load cells to capture the axial load time-history of each horizontal cable, however barrier displacement was not captured. This in turn limits the understanding of the deformation and stiffness behaviour of the flexible barrier during impact. Furthermore, field monitoring is often hindered by difficulties in interpreting results resulting from the inclusion of large boulders, unknown impact orientation, unclear flow properties, and results that are generally unreproducible given the idiosyncrasies of a natural setting (Iverson 2015). 
Extensive small-scale flume modelling has been carried out to investigate the dynamic impact process and the impact load evolution using different flow types. Dry sand flows impacting a mast-like obstacle (Hauksson et al. 2007), rigid barriers (Moriguchi et al. 2009; Choi et al. 2015a; Koo et al. 2016) and baffles (Choi et al. 2014; Ng et al. 2014) have been carried out. Soil and water mixtures impacting rigid barriers and masts have also been carried out (Hübl and Holzinger 2003; Canelli et al. 2012; Scheidl et al. 2013; Cui et al. 2015). However, these studies were carried out at small-scale with limited volume, and without consideration of scaling the stress state.

In recent years geotechnical centrifuge modelling has been widely used to examine debris flow runout and entrainment (Bowman et al. 2010), rock avalanche fragmentation (Bowman et al. 2012), rockfall impact (Chikatamarla et al. 2006), and mobility of dry granular flows under Coriolis effect (Bryant et al. 2015). Centrifuge modelling provides a means to appropriately scale source volume and stresses for flowing sediment problems. The ability to properly characterize the approaching torrent makes the centrifuge a robust tool for studying debris-barrier interaction mechanisms. Given the disparity between the prevalence of rigid and flexible barriers, and the current limited understanding of its impact dynamics, centrifuge modelling appears to be a sensible approach to investigate the landslide flow interaction with barriers and to evaluate existing design approaches. $\mathrm{Ng}$ et al. (2016) reported details of a newly-developed flexible model barrier for modelling debris flow impact in centrifuge. A single preliminary dynamic test using dry Toyoura sand was carried out to verify the performance of the new flexible barrier model under an elevated gravity level of $22.4 \mathrm{~g}$. The dynamic test revealed that the new flexible barrier model was capable of capturing a bilinear 
prototype loading response. However, details of the impact mechanisms, the influence of barrier stiffness and flow properties were not studied and reported.

In this study, systematic centrifuge tests are carried out to examine the impact of dry granular and viscous liquid flows, separately, on both rigid and flexible barriers. The influences of barrier stiffness and materials on impact behaviour are examined.

\section{Centrifuge modelling of barrier impact}

\section{Scaling principles}

Scaling principles for both static and dynamic processes have been well-established over the decades (Schofield 1980; Garnier et al. 1997). Landslide flows are a recent phenomenon to be tested in the geotechnical centrifuge (Bowman et al. 2010) and this study builds on previous works to adopt reasonable scaling laws for the impact problem in this case. Conventional centrifuge scaling laws (Taylor 1995) relevant to this study are summarised in Table 1.

The Froude number $(F r)$ governs the behaviour of open channel granular and viscous flows which are dominated by inertial or gravitational forces, and is given as follows

$F r=\frac{v}{\sqrt{N g h \cos \theta}}$

where $g$ is gravitational acceleration $\left(9.81 \mathrm{~m} / \mathrm{s}^{2}\right), N$ is value in which the gravitational acceleration is elevated, $h$ is the flow depth before impact (m, model scale), and $\theta$ is the channel inclination. It is well-established that the $F r$ governs the impact mechanisms and dynamics (Hübl et al. 2009; Armanini et al. 2011; Choi et al. 2015a).

For the dry granular flow, the Savage number $(N S)$ is adopted in this study to characterise 
the grain shear stresses. Savage number is described by Savage (1984) as the ratio of stress generated via grain collision and contact stresses from the mesoscopic scale. A common form of Savage number is proposed by Iverson $(1997 ; 2015)$

$$
N_{S}=\frac{\not \& \delta^{2}}{g h}
$$

where $\mathcal{\&}$ is shear strain rate $(1 / \mathrm{s}) ; \delta$ is the grain diameter (m). Savage and Hutter (1989) reviewed a variety of experimental evidence and concluded that grain collision stresses dominate if $N s$ is larger than 0.1 , otherwise the frictional (grain contact) stresses dominate.

For the viscous fluid flow, the Reynolds number $(R e)$ is adopted to characterise the ratio of inertial force and viscous force

$$
R e=\frac{\rho_{L} v R_{H}}{\mu_{L}}
$$

where $\rho_{L}$ is the density of viscous liquid $\left(\mathrm{kg} / \mathrm{m}^{3}\right) ; R_{H}$ is the hydraulic diameter of the open channel flow (m); and $\mu_{L}$ is the viscosity of liquid (Pas). Open channel flows characterized with $R e$ larger than 500 are typically turbulent in nature.

\section{Centrifuge impact model}

Tests were carried out at the Geotechnical Centrifuge Facility (GCF) at the Hong Kong University of Science and Technology. The 400 g-ton centrifuge has an arm radius of $4.2 \mathrm{~m}$

(Ng 2014). The tests were performed using a model container with plan dimensions of 1245 $\mathrm{mm} \times 350 \mathrm{~mm}$ and a depth of $851 \mathrm{~mm}$. Fig. 1 shows a side view of the model setup on the centrifuge platform. Inside the model container there is a slope with a channel width of 233 $\mathrm{mm}$ (5.2 $\mathrm{m}$ in prototype) and a length of $1000 \mathrm{~mm}$ (22.4 $\mathrm{m}$ in prototype). The slope is 
inclined at $25^{\circ}$ and supported by an aluminium frame. The Perspex of the model container and a partition are used to form a channelized slope. A storage container with a volume of $0.3 \mathrm{~m}^{3}$ (model scale) was positioned over the slope. The storage container has a hinged door at the bottom and the opening of the door in-flight to release the debris is controlled using a hydraulic actuator.

Fig. 1 shows a flexible barrier rigid post, $200 \mathrm{~mm}$ in height, mounted $530 \mathrm{~mm}(11.9 \mathrm{~m}$ in prototype) from the most upstream end of the slope and flush against the Perspex. The location of the barrier was predetermined by conducting a series of calibration tests to achieve a target $F r$ of about 4.0. The rigid post has ball and socket connections for each horizontal steel strand cable with a diameter of $3.3 \mathrm{~mm}$. In total four horizontal cables, namely top, upper intermediate, lower intermediate, and bottom cable, were used to form the barrier face. The other end of the horizontal cables passed through the partition via pulley systems and were attached to individual spring mechanisms. The spring mechanism comprises one relaxed and one preloaded compression spring in series to model a bilinear loading behaviour of the horizontal cable. A plastic sheet, for simplicity, was applied along the upstream face of the flexible barrier to act as the net to simulate full retention of debris materials during impact. The effective width of flexible barrier is $203 \mathrm{~mm}$ (4.5 $\mathrm{m}$ in prototype). Details of model flexible barrier system are described by $\mathrm{Ng}$ et al. (2016).

Fig. 2 shows the simplified bilinear loading behaviour of a horizontal cable of the model barrier. The dash line shows the load-displacement curve of a prototype flexible barrier cable with energy dissipating elements installed, while the solid line shows the model barrier horizontal cable. The model barrier horizontal cable exhibits a distinct bi-linear loading 
relationship. Before the applied load reaches the inflection point, the slope $K_{1}$ is steep, denoting the elastic stage of a prototype flexible barrier. After reaching the inflection point, stiffness reduces dramatically to model the elongation of energy dissipating elements $\left(K_{2}\right)$. Properties of the prototype flexible barrier system are summarized in Table 2. Typical flexible barriers may have retaining cables linked with the top horizontal cable, this will lead to an obviously stiffer top cable as reflected in the model flexible barrier. Flexible barriers with different stiffness may respond differently under the same impact event. The capacity of a flexible barrier is rated by its energy absorption capacity, not the induced forces inside the barrier components.

A $10 \mathrm{~mm}$ thick steel plate, $200 \mathrm{~mm}$ in height $(4.5 \mathrm{~m}$ in prototype), and $233 \mathrm{~mm}(5.2 \mathrm{~m}$ in prototype) in width was installed to serve as a cantilevered rigid barrier. The ratios between the barrier height and the flow depth of dry sand and viscous liquid are 4.4 and 4.3. The rigid barrier has a bending stiffness $(E I)$ of $1.88 \mathrm{E} 8 \mathrm{Nm}^{2}$ per meter run in prototype (Table 2) and is equivalent to a $0.9 \mathrm{~m}$ thick reinforced concrete wall. The adopted model setup of both flexible and rigid barriers assumes full retention of debris and does not allow for any material to pass through. It is acknowledged that prototype barriers have some degree of permeability and the assumption adopted in this study simplifies the actual impact conditions otherwise observed in the field. However, the assumption of full retention represents a conservative loading scenario for engineering designs. 


\section{Instrumentation}

Nine photoconductive sensors (Silonex NORP12 Cadmium sulphide) were installed along slope centreline at intervals of $50 \mathrm{~mm}(1.1 \mathrm{~m}$ in prototype). Photoconductive sensors are light sensors. When debris covers the photoconductive sensor, a signal is sent to the data logger. With the known time and spacing between sensors, the average frontal velocity can be deduced. The impact process for the initial rigid barrier tests (RS and RL) was captured using a high-speed camera (Prosilica GE 680) with a resolution of $640 \times 480$ pixels sampling at the frequency of 200 frames per second (fps) with a shutter speed $3950 \mu$ s. The velocity attenuation and impact mechanisms behind the barriers can be analysed using Particle Image Velocimetry (PIV) analysis (White et al. 2003; Take 2014). However, it was found that both the resolution and frame rate were insufficient for very accurate PIV analysis, and hence no PIV results are included for tests RS and RL (Figs 3 and 5). Two new high-speed cameras (Mikrotron motionblitz EoSens ${ }^{\circledR}$ mini2, Fig. 1) with improved configurations were used in later flexible barrier tests (FS and FL). The resolution of $1300 \times 1600$ pixels at $640 \mathrm{fps}$ with a short shutter speed $150 \mu$ s proved to be sufficient to capture the impact kinematics for PIV analysis, as shown in Figs 4 and 6 . Illumination was achieved using two $1000 \mathrm{~W}$ LED lights.

Fifteen sets of semiconductor strain gauges (SGs) were mounted to measure the bending moment along the height of rigid barrier (Fig. 7, inset figures). A narrow spacing of $12.5 \mathrm{~mm}$ (0.28 $\mathrm{m}$ in prototype) was chosen so that the dynamic impact pressure of the debris can be derived using double differentiation method ( $\mathrm{Li}$ and Lehane 2010). Load cells were installed along each horizontal cable to measure the induced axial force of the flexible barrier cables. In both the bending moment and axial force measurement, full Wheatstone bridge SGs were 
installed to compensate for temperature effects. The SGs have a gauge factor of 80 and are extremely sensitive. They are capable of measuring strains larger than $1.5 \mu \varepsilon$. An epoxy coating was applied on the surface to protect the SGs. Laser sensors (Wenglor YT44MGV80) with a resolution of $0.2 \mathrm{~mm}$ were used in conjunction with the spring mechanisms of flexible barrier model. Cable displacement and force measurements are captured synchronously. Due to the limited space at the back of the partition, only three laser sensors were utilized to measure the displacement of the top, lower intermediate, and bottom cables.

\section{Test programme and source materials}

Prior to conducting impact tests, a series of open-channel control tests without barriers were carried out (tests CS and CL). A summary of the dynamic characteristics of the flows at the barrier location is given in Table 3. An average $\mathrm{Fr}$ range of about 4.0 was achieved based on the measured velocity and flow depth. Four impact tests using dry sand and viscous fluid on both rigid and flexible barriers were then carried out.

Leighton Buzzard fraction C sand was used in this study and comprises of fairly uniform grains with diameters of about $0.6 \mathrm{~mm}$ (13 $\mathrm{mm}$ in prototype). The pluvial deposition method was used to prepare the sand sample in the storage container. The bulk density of the material held within the storage area is about $1530 \mathrm{~kg} / \mathrm{m}^{3}$. The internal friction angle of the sand is $31^{\circ}$. The interface friction angle of $22.6^{\circ}$ between sand and the channel has been determined by placing the material inside a hollow $5 \mathrm{~cm}$ high cylinder on the base of the flume and then tilting the flume at fractions of one degree until the cylinder slides on the base 
of the flume (Choi et al. 2014).

The viscous liquid used in this series of tests was a mixture of Zinc Chloride $\left(\mathrm{ZnCl}_{2}\right)$, Glycerol, Carboxyl Methyl Cellulose (CMC), and water. Zinc Chloride was adopted for its high solubility under room temperature to increase the liquid density. The final density of the liquid was about $1580 \mathrm{~kg} / \mathrm{m}^{3}$ which is similar with the bulk density of the dry sand. Glycerol was used to reduce the corrosiveness of Zinc Chloride. In order to achieve Froude similarity $(\mathrm{Fr}=4.0)$ with that of natural geophysical flows (Hübl et al. 2009; Cui et al. 2015), it is necessary to reduce the flow velocity in the centrifuge tests. This is achieved by increasing the viscosity of flow fluid (Bowman et al. 2010) using a Carboxyl Methyl Cellulose (CMC) solution. It is noted that CMC solution is a non-Newtonian fluid (Lam et al. 2015) with a shear-thinning behaviour. The use of dry sand and CMC solution is intended to to compare the fundamental impact mechanisms resulting from two simplified flow types, namely frictional and viscous flows. The measured viscosity under the measured shear rate of the flow front is $11.3 \mathrm{~Pa} \cdot \mathrm{s}$ and the viscosity of the subsequent flow increases as the shear rate reduces. It is expected that the rheological behaviour of the viscous liquid used in this study dissipates kinetic energy more efficiently and reaches static conditions quicker than the Newtonian flow. In tests RL and FL, an industrial lubricant with a viscosity of 2.0E-3 Pas, was applied on the Perspex wall to reduce boundary effects.

\section{Testing procedures}

After model preparation, the centrifuge was spun up to $22.4 \mathrm{~g}$. Since the interaction time was scaled down to $1 / 22.4$ of prototype conditions, a sampling rate of $20 \mathrm{kHz}$ was selected to 
capture the details of the dynamic processes. It was ensured that all instruments were stabilized before releasing the debris in-flight via the hydraulic actuator. The dry sand or viscous fluid transitioned on to the slope and impacted the barrier. After which, the centrifuge was spun down.

The flow direction of debris is the same as the centrifuge spin direction (Fig. 1). The Coriolis effect influences the mobility of flowing sediments in a rotating system (Bryant et al. 2015; Bowman et al. 2012). In this study, the Coriolis acceleration reaches up to $85 \%$ of the nominal g-level. However, the influence of the Coriolis acceleration diminishes rapidly once the flow impacts the barrier because the flow velocity rapidly attenuates through grain contact stresses and viscous shearing (Choi et al. 2015a; Koo et al. 2016).

\section{Results of impact tests}

\section{Observed dry granular flow impact mechanisms}

In order to facilitate a comparison, the initial time of all four tests are readjusted to $1.0 \mathrm{~s}$ before the flow front impacts the barrier $(0.045 \mathrm{~s}$ in model scale). Typical interaction durations of the four impact tests were about $0.2 \mathrm{~s}$ in model scale, $4.5 \mathrm{~s}$ in prototype. All subsequent dimensions are presented in prototype unless otherwise stated. Fig. 3 shows a side view of the dry granular impact mechanism on a rigid barrier (test RS) captured using a high speed camera. The flow direction is from left to right. A thin and tapered granular flow front impacted the rigid barrier at $t=1.0 \mathrm{~s}$ with a measured frontal velocity of $13.3 \mathrm{~m} / \mathrm{s} \mathrm{(Fig.} \mathrm{3a).}$ Similarly, a thin layer of run-up developed along the face of the rigid barrier at $t=1.358 \mathrm{~s}$ (Fig. 3b). The granular flow front impact mechanism is consistent with the observed phenomenon 
reported in the experiments conducted by Ashwood and Hungr (2016). The deposition of sand, namely dead zone, occurred at the base of the barrier which further attenuated the subsequent dynamic impact on the barrier (Fig. 3c). Layers of sand continued to pile on top of the dead zone (Fig. 3d). The approaching layers of granular flow became thinner and more deposition is observed (Fig. 3e). The approaching flow eventually ceased to reach and could not impact the barrier at $t=2.800 \mathrm{~s}$ (Fig. 3f) and only deposition further upstream from the barrier occurred (Fig. 3g). The final deposition profile can be observed to form a slope close to the internal frictional angle of the sand. The final deposited flow depth in front of the rigid barrier only reached $55 \%$ of the barrier height.

Fig. 4 shows the dry granular impact mechanism for a flexible barrier (test FS). The impact mechanism is similar to that observed for granular impact against a rigid barrier (test RS) as previously discussed in Fig. 3. The captured snapshot using new high speed camera is shown on the left and its corresponding PIV analysis is shown on the right. The maximum velocity vector is shown as a bold arrow. The flow front with an initial velocity of $13.3 \mathrm{~m} / \mathrm{s}$ impacts the flexible barrier and run-up was observed along the face of the barrier (Fig. 4a). The velocity rapidly decreased by more than $50 \%$ to $6.18 \mathrm{~m} / \mathrm{s}$ upon impact. A distinct dead zone was formed at the base of the flexible barrier as sand continued to layer on top of the ramp-like dead zone (Fig. 4b). The dead zone continued to accumulate and along the upstream direction as sand impacted the barrier (Fig. 4c). The maximum velocity vector appeared at the surface layer and velocity attenuated with depth. The impact process tended towards a static state (Fig. 4d) and the final deposition depth was about $54 \%$ of the barrier height. 
It is apparent from the observed kinematics of this study that before impacting the barrier, the wedge-shaped granular front was in a collisional state. However after impact, the development of a dead zone promoted a predominantly grain-contact state. The Savage number, $N_{\mathrm{S}}$, at $t=1.848 \mathrm{~s}$ (Fig. $4 \mathrm{a}$ ) is estimated as 0.003 at the bottom of flow which implies a contact dominated flow regime. The back-calculated collisional flow regime only existed at a depth of $0.9 \mathrm{~mm}$ from the free surface. Contrary to the results of Jiang and Towhata (2013) and Armanini et al. (2014), clearly distinguished that the inertial and collisional regimes were not observed along the flow depth. This is likely caused by significantly larger particle size adopted in their study (14.1 mm for Jiang and Towhata (2013); $6 \mathrm{~mm}$ for Armanini et al. (2014)).

Observed viscous flow impact mechanisms

Fig. 5 shows the observed viscous liquid impact mechanism for a rigid barrier (test RL). In contrast to thin and tapered dry granular flow fronts, thick and bulbous flow fronts developed for viscous flows. The viscous flow front impacted the barrier at an initial velocity of $12.3 \mathrm{~m} / \mathrm{s}$ (Fig. 5a) and developed much more distinct run-up compared to dry granular flow observed along upstream face of the barrier (Fig. 5c). The run-up or vertical jet (Armanini et al. 2011) was thick and travelled much higher in comparison to that observed for dry granular flow (tests RS and FS). The runup continued to overflow the barrier (Fig. 5d) and tended towards static state at $t=6.608 \mathrm{~s}$ (Fig. $5 \mathrm{~h}$ ) as a free surface began to stabilise. Compared to flume tests carried out by Choi et al. (2015b) using pure water, the viscous liquid did not exhibit a distinct rolling motion back towards the base of the channel. This is attributed to the fact 
that the liquid used in this study has a much higher viscosity. The Reynolds number of the viscous flow before impact is 57 in this study. The flow remained in a laminar state because of the high viscosity of the liquid (for open channel laminar flows $R e<500$ ).

Fig. 6 shows the viscous liquid impact mechanism against a flexible barrier (test FL). The flow front impacted the flexible barrier with an initial velocity of $12.3 \mathrm{~m} / \mathrm{s}$ and rapidly decelerated to $9.78 \mathrm{~m} / \mathrm{s}$ (Fig. 6a). A thick runup is then observed (Fig. 6b), however a lower runup height and no distinct overflow occurred as the deformation of the flexible barrier attenuated the flow energy and provided a larger retention volume. The lobe of viscous liquid along the face of the barrier continued to increase in thickness (Fig. 6c) and eventually tended towards a static state (Fig. 6d). The viscous liquid exhibited a $20 \%$ reduction in velocity compared with $50 \%$ for granular flow impacting a flexible barrier (test FS) after impact. The compressibility of granular flow leads to greater energy dissipation during the impact process. A comparison of the maximum velocity vectors during the impact sequence for dry granular flow and viscous fluid shows that for granular flow, the maximum velocity vectors were observed at the free surface of the flow, whereas the viscous liquid generally exhibited its maximum vectors around the base of the vertical jet except when the liquid was tending towards a static state. No dead zone was observed at the base of flexible barrier. It is also worthwhile to mention that large vectors were observed in the upstream direction for the viscous liquid tests (Fig. 6b) compared to the granular flow tests. This is indicative of a large impact pressure from significant change in momentum. 
Rigid barrier bending moment

Figs.7a and $7 \mathrm{~b}$ show the development of measured bending moment per unit width for granular (test RS) and viscous liquid impact (test RL) on the rigid barrier using strain gauges, respectively. In Fig. 7a, the initial response of the barrier upon sand flow impact was recorded at $t=1.0 \mathrm{~s}$, after which a slight peak was captured at $t=2.8 \mathrm{~s}$, and the impact process ended at $t=3.0 \mathrm{~s}$. As discussed earlier, sand only deposited along $55 \%$ of the barrier (Fig. 3), hence only strain gauges with registered measurements are shown. The bending moment captured at different elevations all exhibited a slight peak, and as expected, the largest bending moment was captured at the base of the barrier (SG 1).

For viscous liquid impacting against a rigid barrier (Fig. 7b), the measured impact process commenced at $t=1.0 \mathrm{~s}$, and a much more noticeable peak was observed at $t=2.8 \mathrm{~s}$, after which impact process did not reach a static state until after $t=6.0 \mathrm{~s}$. A comparison of bending moment development between granular and viscous liquid flow shows that the impact duration, before reaching a static state, is longer for viscous liquid. Similar to granular impact against a rigid barrier (test RS), the maximum bending moment was measured at SG 1 at the barrier base. Viscous liquid exhibited bending moments at SG 1 almost 7 times that of granular flow. A theoretical static line is drawn in Fig. $7 \mathrm{~b}$ to compare with the measured bending moment at SG 1. The differences observed between granular and viscous liquid flow against a rigid barrier is mainly attributed to the intrinsic properties of the two flow media. The results from this study only pertain to simplified flow cases, i.e., dry sand and viscous liquid flows. Caution should be taken when extrapolating the results for natural geophysical flows. 
Fig. 8 shows the evolution of bending moment along the depth of the barrier for viscous liquid impact against the rigid barrier (test RL). The bending moment profiles with depth are shown before run-up has reached the crest of the barrier $(t=1.1 \mathrm{~s})$, at the peak bending moment $(t=2.8 \mathrm{~s})$, during overflow $(t=4.4 \mathrm{~s})$, and at a static state $(t=124.1 \mathrm{~s})$. If the barrier is regarded as a cantilever beam, then it is expected that during the loading process behind the barrier, the bending moment would be highly non-linear in nature. However, once overflow occurred $(t=2.8 \mathrm{~s})$, the bending moment profile would tend towards a more linear profile since a drag force is induced at the crest of the barrier from overflow. While the bending moment caused by the impact pressure acting on lower position is shielded by the concentrated force effect.

Flexible barrier axial cable force and displacement

Figs. 9a and 9b show time histories for granular impact induced axial cable forces and displacements for test FS, respectively. Loading first occurred in the bottom cable and as sand deposited behind the barrier (Fig. 9a), and then captured in the lower and upper intermediate cables about $0.5 \mathrm{~s}$ and $0.9 \mathrm{~s}$ after the bottom cable, respectively. Given that the deposition height of the sand was only $54 \%$ of the barrier height, the top and intermediate cables only registered forces of less than $20 \mathrm{kN}$. It is worthwhile to mention that each cable exhibits a not very distinct peak axial load. During the initial loading stage, both the bottom and lower intermediate cables exhibited a sudden drop in load. This drop could be attributed to a change in stiffness because the loading reached the inflection point (Fig. 2). The inflection point indicates the activation of the energy dissipating elements in a prototype 
barrier. It is also apparent that after the change in stiffness, the cable showed a slower rate of loading and a higher degree of fluctuation.

The corresponding cable displacement of granular impact on a flexible barrier (test FS) is shown in Fig. 9b. Given the constraint of space on the back of the partition, only three laser sensors were installed to capture the displacement of three horizontal cables, namely the bottom, lower intermediate, and top cables. Upon impact, the cable displacements rapidly increased until a static state was reached. The largest displacement is observed in the bottom cable, which also coincides with the largest measured axial load (Fig. 9a). Decreasing displacement is observed with increasing height along the flexible barrier. Almost no displacement was registered in the top cable. A maximum elongation of $0.5 \mathrm{~m}$ (prototype) was captured for the bottom cable.

Figs. 10a and 10b show the cable load and displacement time histories for viscous liquid impact on the flexible barrier (test FL), respectively. The cable force exhibited distinct peak loads in each cable. The bottom and lower intermediate cables were loaded at about the same instant which can be explained by the observed vertical jet bulge that rapidly ran up along the barrier. The peak load captured in the top cable confirms that the flow front climbed up to the crest of barrier (Fig. 6). Moreover, minor drops in measured loading are attributed to the loading behaviour reaching the inflection point (Fig. 2) and transitioning to a change in stiffness in each cable. The cable elongation of test FL is shown in Fig. 10b. Measurement shows a maximum elongation of about $1 \mathrm{~m}$ (prototype) in the bottom cable. 


\section{Comparison of total loads and pressure}

Pressure evolution on rigid barrier

The impact pressure distribution along the rigid barrier and the total impact load are of the most interest to designing a debris-resisting structure. By conducting double differentiation (Li and Lehane 2010; Rollins et al. 2005) of the bending moment, the pressure distribution along the rigid barrier can be deduced. Fig. 13 shows the evolution of viscous flow pressure distribution along the depth of the barrier. Similarly, the pressure distributions are shown before overflow, at the peak bending moment, during overflow, and at static conditions. At the initial stage of impact ( $t=1.1 \mathrm{~s}$ ), pressure is only induced on the bottom half of the barrier with a relatively linear distribution. As overflow occurred ( $t=2.8 \mathrm{~s}$ and $4.4 \mathrm{~s})$, the pressure distribution became highly non-linear, and once the debris reaches a static state, the pressure distribution could become linear again. Along the bottom half of the barrier, or about 2 times the approach flow depth, the pressure significantly exceeded static conditions. Whereas along the top half of the barrier, flow velocity vectors were generally flush with the barrier face during interaction, and the dynamic pressure was bounded within the static envelope. It is apparent from the pressure distribution profiles that majority of the momentum flux, which is perpendicular to the barrier face, is resisted by the bottom 2-times approach depth of the barrier. A similar observation was reported by Armanini et al. (2011) who adopted pressure gauges to measure the pressure distribution caused by the impact jet along a rigid barrier. 


\section{Comparison of peak and static loads}

The impact load on each cable and summation of loads from all four cables (resultant) have been derived. The impact loads shown in Figs. 11 and 12 are perpendicular to the barrier face and not axial cable forces which are along the cable direction. Details of the derivation of the impact load from the axial cable force and displacement are described in Appendix. Figs. 11a and $11 \mathrm{~b}$ show the evolution of flexible barrier impact load and distribution with depth for granular impact (test FS). The trends of the impact load are quite similar with those in axial cable force, yet the magnitudes are much higher. From $t=1.2 \mathrm{~s}$ to $t=1.9 \mathrm{~s}$, the bottom cable impact load only increased from about $50 \mathrm{kN}$ to $175 \mathrm{kN}$ (Fig. 11b). The impact load distribution on the four cables is nonlinear and the bottom cable took more than half $(71 \%)$ of the resultant load at static condition. Figs. $12 \mathrm{a}$ and $12 \mathrm{~b}$ show the evolution of flexible barrier impact load with depth for viscous flow impact (test FL). The total impact load after $\mathrm{t}=6.6 \mathrm{~s}$ (static value) of test FL is further compared with the theoretical value. The rate of loading upon impact for viscous flows (test FL) increased quite substantially from $t=1.2 \mathrm{~s}$ to $t=1.9 \mathrm{~s}$, and the loading from the bottom cables rapidly increased from about $50 \mathrm{kN}$ to 375

kN (Fig. 12b). For test FL, the bottom cable took $58 \%$ of the total load at static condition.

Furthermore, a comparison between the impact load evolution for granular and viscous flows shows that viscous flows could generate much higher impact loads. As aforementioned, this is attributed to the intrinsic differences in material properties of viscous and granular flows. The observed impact mechanisms can be correlated and used to explain why obvious loading was picked up for viscous flow impact along the top cable (test FL). Viscous flows develop a vertical bulge mechanism (Fig. 6) that runs-up along the vertical face 
of the barrier with greater ease compared to granular flow which piles-up in layers and compresses behind the barrier (Fig. 4). The impact mechanism for granular flow suppresses run-up as it is efficient in absorbing impact energy, hence load was not generated in the top cable for granular flow impact (test FS).

The resultant loads in terms of peak and static values of the four tests are summarized in Table 4. The total impact loads of test RS and RL are achieved by integrating the impact pressure along depth. The peak impact load of test RS is only $57 \%$ of the peak load of test RL, while peak impact load of test FS only reaches $41 \%$ of test FL. The peak impact load of test FS is only $56 \%$ of that of test RS, while peak impact load of test FL reaches $80 \%$ of that of test RL. For test RL and FL, the static free surface is horizontal and the static loads are close with each other. For test RS and FS, there are similar deposition heights but different loads. The static load of test FS is $39 \%$ lower than that of test RS, and the cause will be discussed in the next section.

Comparison of static earth pressure

The static pressures behind the rigid and flexible barriers are further compared to examine the state of deposited materials (Fig. 14). As both rigid and flexible barriers tend to deflect downstream upon loading, the pressure coefficient of the granular material is expected to be close to the active pressure coefficient $\left(k_{\mathrm{a}}\right)$. Given the inclined barrier and sloping free surface of dry sand, the $k_{\mathrm{a}}$ value based on Coulomb theory is as high as 1.7. For test RS, the deduced earth pressure coefficient is 2.3 , which implies that the sand had not been fully mobilized to the active mode. The deduced static earth pressure coefficient of test FS was 1.5 and is lower 
than the theoretical $k_{a}$. As sand progressively deposited behind flexible barrier during impact, the incremental increase in lateral earth pressure triggered the elongation of the energy dissipating devices. The lateral deformation of flexible barrier in turn fully mobilized the internal strength of the deposited debris, allowing it to reach an active failure mode. This ensures a lower lateral earth pressure acting on the flexible barrier ( $\mathrm{Ng}$ et al. 2016). Furthermore, the impact on a flexible barrier is not entirely a two-dimensional problem. Arching effects (Handy 1985; Iglesia et al. 2013) on the rigid post likely causes the deduced earth pressure coefficient to be lower than the theoretical value. For viscous liquid, the deduced pressures coincide with the theoretical value (1.1). This in turn reflects that the total load and static pressure difference in test RS and FS originates from the different soil states behind the deflected barriers, rather than from errors attributed to instruments and interpretation.

\section{Influence of barrier stiffness and material types}

Although large scale tests have been carried out to study the impact load on flexible barriers (Duffy and DeNatale 1996; Wendeler et al. 2007; Bugnion and Wendeler 2010; Kwan et al 2014), less attention has been paid on a direct comparison of the stiffness effect between rigid and flexible barriers. Rigid barriers can be regarded as flexible barriers with extremely high stiffness. The results from both rigid and flexible barrier impact tests reveal that: (1) flexible barrier cable elongation provides an enlarged retention volume. This results in the full retention of the debris and reduced run-up as observed in test FL, (2) longer interaction duration during flexible barrier impact results in a drop of peak load from $884 \mathrm{kN}$ to $704 \mathrm{kN}$ 
from test RL to test FL, and (3) soil arching in test FS could lead to earth pressures even lower than the active earth pressure.

Two distinct interaction patterns for granular and viscous fluid flows have been observed. For tests pertaining to granular impact (tests RS and FS), the granular front forms an inert mass upon initial impact (Fig. 4) which shields the structure from subsequent loading (Hungr et al. 1984). Viscous flow impact (tests RL and FL) exhibits a vertical jet-like bulge (Fig. 6). This generates a further thrust surpassing the one caused by the first impact of the front and is consistent with findings reported by Canelli et al. (2012). In addition, the upstream velocity vector captured from PIV analysis is indicative of a much higher induced impact load compared to that of sand (Figs. 6a and 6b).

Besides the distinct interaction patterns for granular and viscous fluid flows, distinct loading behaviours are also observed. Comparisons of rigid and flexible barrier impact for granular flows (tests RS and FS) and viscous fluid flows (tests RL and FL) indicate a drop in peak load of $44 \%$ (arching included) and 20\% respectively. On the contrary, changes in material types for the rigid barrier (tests RL and RS) and flexible barrier (tests FL and FS) reveal a drop in peak load up to $43 \%$ and $59 \%$, respectively. The intrinsic property of granular flow allows a portion of the momentum flux to be resisted by the inter-granular friction, whereas the momentum flux of incompressible viscous fluid flow could be more readily transferred to the barrier. There is a strong indication from this study that characterisation of the flow type should have a great significance when designing structural countermeasures. 


\section{Comparison of impact pressure based on hydrodynamic approach}

The hydrodynamic approach (Eqn. 1) is adopted to back-calculate the dynamic pressure coefficient $\alpha$ for each test in this study and the deduced $\alpha$ values are compared with the experimental or field monitoring results of the previous reseachers (Cui et al. 2015; Hübl and Holzinger 2003; Proske et al. 2011; Tiberghien et al. 2007; Zhang and Yuan 1985) in Fig. 15. It is acknowledged that a typical impact process comprises both dynamic and static loading. However, only $\alpha$ values corresponding to the dynamic or frontal impact, without obvious debris deposition, have been calculated. The frontal flow depth $h$ of dry sand is defined as the depth where the sand front impacted the barrier before an obvious dead zone formed. To account for the uncertainty of the measured flow velocity and thickness, error bars are illustrated. The error bars are estimated relative to the average values measured in the experiments. The minimum and maximum values are summarized in Table 5.

Each experiment or prototype event shown only characterizes a narrow range of specific $\mathrm{Fr}$, thus a comparison of $\alpha$ values across a broad spectrum of dynamic conditions can serve to bear additional insight on the relevance of the tests carried out in this study. Dry granular flow fronts will naturally develop a wedge-shaped profile, hence higher $F r$ is captured compared to the overall $\mathrm{Fr}$ listed in Table 3. The deduced $\alpha$ values in this study during frontal impact are around unity, regardless of barrier type. A theorectical value of unity characterizes the pressure coefficient for a liquid based on the conservation of momentum. The reason for a lack of dependency of $\alpha$ values on material type is attributed to a relatively large barrier stiffness compared to the compliant flow medium. However, it is imperative to bear in mind that boulder impact is not captured in this study. It is expected that the bouldery front can 
impose much larger impulse loads on a structure, due to the high stiffness of the boulder and short interaction time (Cui et al. 2015; Kwan et al. 2013; Scheidl et al. 2013). A systematic investigation of the influence of boulder size and distribution is necessary to bear additional insight on the hydrodynamic approach.

From the comparison, $\alpha$ value drops rapidly as the $F r$ increases when $F r$ is between 0 and 2 . As the $F r$ increases above 2, there is less dependency of $\alpha$ coefficient on $F r$ conditions. At the low $F r$ range, the impact force is dominated by the static load. If the hydrodynamic load model is adopted under such condition, the $\alpha$ value has the following expression

$$
\alpha=\frac{0.5 \kappa\left(\rho g h^{2} w\right)}{\rho v^{2} h w}=\frac{0.5 \kappa}{F r^{2}}
$$

the hydrostatic coeffcient $\kappa$ here is not determined and is reported to reach up to 50 (Hübl et al. 2009). In Eqn. 6, a low $F r$ value results in a high $\alpha$ value for the hydrodynamic method. Current design guidelines suggest constant dynamic/static pressure coefficients for debris-resiting structure design. While Hübl et al. (2009) recommends adopting the $F r$ as a criterion for using either the hydrostatic and hydrodynamic methods. With $F r$ higher than 2, hydrodynamic models work quite well, while hydrostatic models are more relevant for low $F r$. In this study, all the $\alpha$ values could be well below 2, where 2 is the recomended value for flexible barrier design (WSL 2009; Kwan et al. 2013). It appears that $\alpha$ value higher than 2 for $\mathrm{Fr}$ lower than 4, the deduced impact load could be overly conservative if boulder impact is neglected. 


\section{Energy dissipation and debris barrier interaction}

Energy absorption of each flexible barrier impact test can be estimated based on measured cable force and displacement. For test FS, the flexible barrier stored $73 \mathrm{~kJ}$ of the sand kinetic energy. In contrast, for viscous fluid flow, $249 \mathrm{~kJ}$ of the barrier energy capacity was mobilized (test FL). Although the degree of flexible barrier energy utilisation was different for granular and viscous flows, both flow cases were successfully intercepted by the flexible barrier. Nevertheless, the initial bulk potential energy of the debris materials in the storage container before releasing on the slope was about $10 \mathrm{MJ}$ (prototype). It is evident that the bulk energy of the debris material was not dissipated by the flexible barrier modelled in the centrifuge test. Instead, the debris-barrier interaction facilitates the dissipation of energy in the flow mass. The intrinsic properties of the debris materials could play an important role in the dissipation process. Boundary and internal shearing of the debris medium is instrumental in dissipating the bulk initial energy.

Debris-resisting flexible barrier originates from the rockfall barrier, but the energy dissipation and momentum resisting mechanisms are quite different. Boulders, usually several meters in diameter, imposes a concentrated load on the barrier face. The impulse results in localised deformation and the kinetic energy is mainly dissipated by the barrier itself. In contrast, landslide debris interact with the flexible barrier and results in attenuation of the energy within the flow. The debris front imposes impulse load on the barrier with a spread area (Canelli et al. 2012) and the subsequent flow is influenced by the interaction and interacts with both the previous sediments and barrier in a relatively mild manner. The 
results from this study show why a landslide debris event with an energy level several orders of magnitude higher can be intercepted by a flexible barrier with a much lower magnitude of energy dissipating capacity. Findings from this study are consistent with research outcomes from Moriguchi et al. (2009), Ashwood and Hungr (2016), Koo et al. (2016), and GEO (2016), suggesting that the dynamic force induced on an obstacle by dry granular flow is mainly attributed to the inertial effect of the frontal region. The momentum after frontal impact is attenuated by the internal friction on the deposited mass (dead zone). Current design practice neglecting the effect of dead zone formation and run-up may lead to conservative estimates of impact loads. The guideline recommended by GEO (2016) could be a sensible means to allow consideration of debris-barrier interaction in design. Nevertheless, in real life, debris flows could comprise hard inclusions (e.g. boulders) which are not considered in this study. The use of a higher $\alpha$ value could still be warranted.

\section{Summary and conclusions}

Both dry granular flow and viscous liquid impact tests against rigid and flexible barriers were carried out using the geotechnical centrifuge. Findings from this study are given as follows

a) Characterisation of the flow type could have a significance when designing structural countermeasures. The stress-dependent shear strength of sand facilitates the formation of a dead zone as sand progressively layers on top and impacts the barrier. The stress-independent strength and incompressible nature of viscous liquid flow is instrumental for the observed vertical-jet impact mechanism. PIV analysis results have 
shown an upstream component of the velocity vector in viscous liquid, implying a higher impact load than dry sand on the barriers. A change in material type from viscous to granular for test FL and FS causes a drop up to $59 \%$ in peak impact load.

b) During the frontal impacts of dry granular and viscous flow, the dynamic pressure coefficients of hydrodynamic approach are close to unity, regardless of barrier type. This is because the stiffness of both the rigid and flexible barriers are much higher than the bulk stiffness of the single-phase flows in this study. The bulk stiffness of the flows may be increased by the addition of boulder entrainment.

c) Debris-resisting flexible barriers rely on the interaction between the flow media with intrinsic properties and the barriers to arrest the entire torrent. The barrier only arrests the flow front and relies on the development of distinct impact mechanisms to dissipate the majority of the debris kinetic energy. This denotes the main difference between debris-resisting flexible barrier and rockfall flexible barrier.

d) Based on the back-analysis of static earth pressure coefficients of test RS and FS, it is found that the total load difference originates from the different modes of the dry sand. The large deformation of flexible barrier ensures an active failure mode and minimum lateral load on the structure.

Only single phase (dry sand and viscous liquid) flows impact was investigated in this study. However, they cannot capture the key feature of real debris flows: solid-fluid interaction. The evolving debris dilation rate coupled with the evolution of pore pressure plays a primary role in regulating debris flow dynamics (Iverson and George 2014, 2016). The coupling effect of the solid and fluid implies a much complicated impact of two phase mixture (Armanini et al. 
2014; Hungr et al. 2014). Further investigation of the solid-fluid mixture impact on rigid and

flexible barriers is necessary.

\section{Acknowledgements}

The authors are grateful for financial support from research grant $\mathrm{T} 22-603 / 15 \mathrm{~N}$ provided by the Research Grants Council of the Government of Hong Kong SAR, China and the HKUST Jockey Club Institute of Advanced Study for their support. The support by the Geotechnical Engineering Office, Civil Engineering and Development Department of the Government of the Hong Kong SAR is gratefully acknowledged. This paper is published with the permission of the Head of the Geotechnical Engineering Office and the Director of Civil Engineering and

Development, the Government of the Hong Kong SAR.

\section{References}

Armanini, A., Larcher, M., Nucci, E., and Dumbser, M. 2014. Submerged granular channel flows driven by gravity. Advances in Water Resources, 63, 1-10.

Armanini, A., Larcher, M., and Odorizzi, M. 2011. Dynamic impact of a debris flow front against a vertical wall. In Proceedings of the 5th International Conference on Debris-Flow Hazards Mitigation: Mechanics, Prediction and Assessment, Padua, Italy. pp. 1041-1049.

Ashwood, W., and Hungr, O. 2016. Estimating the total resisting force in a flexible barrier impacted by a granular avalanche using physical and numerical modeling. Canadian Geotechnical Journal, doi: 10.1139/cgj-2015-0481.

Bowman, E.T., Laue, J., Imre, B., and Springman, S.M. 2010. Experimental Modelling of Debris Flow Behaviour Using a Geotechnical Centrifuge. Canadian geotechnical journal, 47(7): 742-762.

Bowman, E.T., Take, W.A., Rait, K.L., and Hann, C. 2012. Physical Models of Rock Avalanche Spreading Behaviour with Dynamic Fragmentation. Canadian Geotechnical 
Journal, 49(4): 460-476.

Bryant, S., Take, W., Bowman, E., and Millen, M. 2015. Physical and numerical modelling of dry granular flows under Coriolis conditions. Géotechnique, 65(3): 188-200.

Bugnion, L., and Wendeler, C. 2010. Shallow landslide full-scale experiments in combination with testing of a flexible barrier. In Monitoring, Simulation, Prevention and Remediation of Dense and Debris Flows III, pp 161-173.

Canelli, L., Ferrero, A.M., Migliazza, M., and Segalini, A. 2012. Debris Flow Risk Mitigation by the Means of Rigid and Flexible Barriers - Experimental Tests and Impact Analysis. Natural Hazards and Earth System Science, 12(5): 1693-1699.

Chikatamarla, R., Laue, J., and Springman, S.M. 2006. Centrifuge Scaling Laws for Guided Free Fall Events Including Rockfalls. International Journal of Physical Modelling in Geotechnics, 6(2); 15-26.

Choi, C.E., Au-Yeung, S.C.H., Ng, C.W.W., and Song, D. 2015a. Flume investigation of landslide granular debris and water runup mechanisms. Géotechnique Letters, 5: 28-32.

Choi, C.E., Ng, C.W. W., Au-Yeung, S.C.H., and Goodwin, G. 2015b. Froude characteristics of both dense granular and water flows in flume modelling. Landslides, 12(6), 1197-1206.

Choi, C. E., Ng, C.W.W., Song, D., Kwan, J.H.S., Shiu, H.Y.K., Ho, K.K.S., and Koo, R.C.H. 2014. Flume Investigation of Landslide Debris-Resisting Baffles. Canadian Geotechnical Journal, 51(5): 540-553.

Cui, P., Zeng, C., and Lei, Y. 2015. Experimental analysis on the impact force of viscous debris flow. Earth Surface Processes and Landforms. doi:10.1002/esp.3744

Duffy, J.D., and DeNatale, J.S. 1996. Debris Flow Mitigation Using Flexible Barriers. Proceedings of the 47th Annual Highway Geology Symposium, pp. 243-252.

Garnier, J., Gaudin, C., Springman, S.M., Culligan, P.J., Goodings, D., Konig, D. and Thorel, L. 2007. Catalogue of scaling laws and similitude questions in geotechnical centrifuge modelling. International Journal of Physical Modelling in Geotechnics, 7(3), 1.

GEO. 2016. Assessment of Landslide Debris Impact Velocity for Design of Debris-resisting Barriers. Technical Guidance Note No. 44 (TGN 44). Geotechnical Engineering Office, Civil Engineering Department of CEDD, HKSAR. 
Handy, R. 1985. The Arch in Soil Arching. J. Geotech. Engrg. 111(3), 302-318.

Hauksson, S., Pagliardi, M., Barbolini, M., and Jóhannesson, T. 2007. Laboratory measurements of impact forces of supercritical granular flow against mast-like obstacles. Cold Regions Science and Technology, 49(1), 54-63.

Hübl, J., Suda, J., Proske, D., Kaitna, R., and Scheidl, C. 2009. Debris flow impact estimation. In Proceedings of the 11th International Symposium on Water Management and Hydraulic Engineering, Ohrid, Macedonia. pp. 1-5.

Hübl, J., Holzinger, G., and Wehrmann, H. 2003. Entwicklung von Grundlagen zur Dimensionierung kronenoffener Bauwerke für die Geschiebebewirtschaftung in Wildbächen: Klassifikation von Wildbachsperren, WLS Report 50. Im Auftrag des BMLFUW VC 7a (unveröffentlicht). Wien: Institut für Alpine Naturgefahren, Universität für Bodenkultur.

Hungr, O., Morgan, G. C., and Kellerhals, R. 1984. Quantitative analysis of debris torrent hazards for design of remedial measures. Canadian Geotechnical Journal, 21(4), 663-677.

Hungr, O., Leroueil, S., and Picarelli, L. 2014. The Varnes classification of landslide types, an update. Landslides, 11(2), 167-194.

Iglesia, G., Einstein, H., and Whitman, R. 2013. Investigation of Soil Arching with Centrifuge Tests. J. Geotech. Geoenviron. Eng., 10.1061/(ASCE)GT.1943-5606.0000998, 04013005

Iverson, R. M. 1997. The physics of debris flows. Reviews of geophysics, 35(3): 245-296.

Iverson, R. M., and George, D. L. 2014. A depth-averaged debris-flow model that includes the effects of evolving dilatancy. I. Physical basis. In Proceedings of the Royal Society of London A: Mathematical, Physical and Engineering Sciences 470, No. 2170, 20130819.

Iverson, R.M. 2015. Scaling and design of landslide and debris-flow experiments. Geomorphology 244, 9-20.

Iverson, R.M. and George, D.L. 2016. Modelling landslide liquefaction, mobility bifurcation and the dynamics of the 2014 Oso disaster. Géotechnique 66, No. 3, 175-187. 
Jiang, Y.J., and Towhata, I. 2013. Experimental study of dry granular flow and impact behavior against a rigid retaining wall. Rock mechanics and rock engineering, 46(4): 713-729.

Koo, R.C.H., Kwan, J.S.H., Ng, C.W.W., Lam, C., Song, D. and Pun, W. K.. 2016. Velocity attenuation of debris flows and a new momentum-based load model for rigid barriers. Landslides. doi:10.1007/s10346-016-0715-5.

Kwan, J.S.H. 2012. Supplementary Technical Guidance on Design of Rigid Debris-resisting Barriers. GEO Report No. 270. Geotechnical Engineering Office, HKSAR Government.

Kwan, J.S.H., Koo, R.C.H. and Ko, F.W.Y. 2013. A Pilot Study on the Design of Multiple Debris-resisting Barriers. Technical Note No. TN 3/2013. Geotechnical Engineering Office, Civil Engineering Department of CEDD, HKSAR.

Kwan, J.S.H., Chan, S.L., Cheuk, J.C. ., and Koo, R.C.H. 2014. A case study on an open hillside landslide impacting on a flexible rockfall barrier at Jordan Valley, Hong Kong. Landslides, 11(6): 1037-1050.

Lam, C., Martin, P. J., and Jefferis, S. A. (2015). Rheological properties of PHPA polymer support fluids. Journal of Materials in Civil Engineering, 27(11), 04015021.

Li, A.Z., and Lehane, B.M. 2010. Embedded cantilever retaining walls in sand. Géotechnique, 60(11): 813-823.

McArdell, B.W., Bartelt, P., and Kowalski, J. 2007. Field observations of basal forces and fluid pore pressure in a debris flow. Geophysical Research Letters, 34(7).

Moriguchi, S., Borja, R.I., Yashima, A., and Sawada, K. 2009. Estimating the impact force generated by granular flow on a rigid obstruction. Acta Geotechnica, 4(1): 57-71.

Ng, C.W.W. 2014. The state-of-the-art centrifuge modelling of geotechnical problems at HKUST. Journal of Zhejiang University SCIENCE A 15(1): 1-21.

Ng, C.W.W., Song, D., Choi, C.E., Koo, R.C.H., and Kwan, J.S.H. 2016. A newly developed flexible barrier system for modelling of landslide debris impact in centrifuge. Géotechnique Letters (accepted)

Proske, D., Suda, J., and Hübl, J. 2011. Debris flow impact estimation for breakers. Georisk, $5(2), 143-155$.

Rollins, K.M., Gerber, T.M., Lane, J.D., and Ashford, S.A. 2005. Lateral resistance of a 
full-scale pile group in liquefied sand. Journal of Geotechnical and Geoenvironmental Engineering, 131(1), 115-125.

Savage, S.B. 1984. The mechanics of rapid granular flows. Adv. Appl. Mech. 24, 289-366.

Savage, S.B., and Hutter, K. 1989. The motion of a finite mass of granular material down a rough incline. Journal of liquid mechanics, 199, 177-215.

Scheidl, C., Chiari, M., Kaitna, R., Müllegger, M., Krawtschuk, A., Zimmermann, T., and Proske, D. 2013. Analysing debris-flow impact models, based on a small scale modelling approach. Surveys in Geophysics, 34(1): 121-140.

Take, W.A. 2014. Thirty-sixth Canadian Geotechnical Colloquium: advances in visualization of geotechnical processes through digital image correlation. Canadian Geotechnical Journal. doi:10.1139/cgj-2014-0080.

Taylor, R.N., ed. 1995. Geotechnical Centrifuge Technology, 1st Ed., Blackie Academic Professional, Glasgow, U.K.

Tiberghien, D., Laigle, D., Naaim, M., Thibert, E., and Ousset, F. 2007. Experimental investigation of inter-action between mudflow and obstacle. Debris-flow hazards mitigation: mechanics, prediction and assessment, Millpress, Rotterdam.

Volkwein, A., Schellenberg, K., Labiouse, V., Agliardi, F., Berger, F., Bourrier, F., Dorren, L. K. A., Gerber, W., and Jaboyedoff, M. 2011. Rockfall Characterisation and Structural Protection-a Review. Natural Hazards and Earth System Sciences, 11: 2617-2651.

Wendeler, C., Volkwein, A., Denk, M., Roth, A., and Wartmann, S., 2007. Field Measurements Used for Numerical Modelling of Flexible Debris Flow Barriers, In: CL Chen, JJ Major (eds). Proceedings of Fourth International Conference on Debris Flow Hazards Mitigation: Mechanics, Prediction, and Assessment, Chengdu, China, 10-13 September 2007. pp. 681-687.

White, D.J., Take, W.A., and Bolton, M.D. 2003. Soil deformation measurement using particle image velocimetry (PIV) and photogrammetry. Geotechnique, 53(7): 619-631. 
WSL. 2009. Full-scale Testing and Dimensioning of flexible debris flow barriers. Technical report 1-22. WSL, Birmensdorf.

Zhang, S., and Yuan, J. 1985. Impact force of debris flow and its detection. Memoirs of Lanzhou Institute of Glaciology and Cryopedology, Chinese Academy of Sciences. Beijing: Science Press, 269-274. 


\section{Figure Captions}

Fig. 1 Side view of model setup and instrumentation.

Fig. 2 Load-displacement behaviour of flexible barrier bottom cable (all dimensions in prototype) (Ng et al. 2016).

Fig. 3 Observed interaction kinematics for test RS in prototype time: (a) $t=1.0 \mathrm{~s}$; (b) $t=$ $1.358 \mathrm{~s}$; (c) $t=1.725 \mathrm{~s}$; (d) $t=2.083 \mathrm{~s}$; (e) $t=2.441 \mathrm{~s}$; (f) $t=2.800 \mathrm{~s}$; (g) $t=3.158 \mathrm{~s}$; (h) $t=$ $4.771 \mathrm{~s}$. The camera field of view was adjusted flush with the slope $\left(25^{\circ}\right)$.

Fig. 4 Observed interaction kinematics for test FS in prototype time: (a) $t=1.848 \mathrm{~s}$; (b) $t=$ $2.128 \mathrm{~s}$; (c) $t=2.520 \mathrm{~s}$; (d) $t=3.248 \mathrm{~s}$. The bold arrows denote the maximum velocity in each figure.

Fig. 5 Observed interaction kinematics for test RL in prototype time: (a) $t=1.0 \mathrm{~s}$; (b) $t=$ $1.232 \mathrm{~s}$; (c) $t=1.568 \mathrm{~s}$; (d) $t=2.016 \mathrm{~s}$; (e) $t=2.464 \mathrm{~s}$; (f) $t=2.912 \mathrm{~s}$; (g) $t=3.360 \mathrm{~s}$; (h) $t=$ $6.608 \mathrm{~s}$. The camera field of view was adjusted flush with the slope $\left(25^{\circ}\right)$.

Fig. 6 Observed interaction kinematics for test FL in prototype time: (a) $t=2.408 \mathrm{~s}$; (b) $t=$ $2.688 \mathrm{~s}$; (c) $t=3.248 \mathrm{~s}$; (d) $t=3.808 \mathrm{~s}$. The bold arrows denote the maximum velocity in each figure.

Fig. 7 Bending moment time history of (a) test RS and (b) test RL (unit width)

Fig. 8 Bending moment evolution for test RL (unit width)

Fig. 9 (a) cable force time history for test FS (b) cable displacement time history for test FS.

Fig. 10 (a) cable force time history for test FL (b) cable displacement time history for test FL.

Fig. 11 (a) resolved impact load time history and (b) impact load evolution for test FS.

Fig. 12 (a) resolved impact load time history and (b) impact load evolution for test FL.

Fig. 13 Impact pressure evolution for test RL.

Fig. 14 Static pressure acting on rigid and flexible structures induced by dry sand and liquid. The barrier is $25^{\circ}$ inclined from vertical direction.

Fig. 15 Relationship between $\alpha$ and Fr. Except the flexible barrier data points, all the others are from rigid structure tests. 


\section{Tables}

Table 1. Relevant scaling laws

\begin{tabular}{ccc}
\hline Parameter & Dimension & $\begin{array}{c}\text { Scaling law } \\
\text { (model / prototype) }\end{array}$ \\
\hline Gravity & $L / T^{2}$ & $N$ \\
Density & $M / L^{3}$ & 1 \\
Length (displacement) & $L$ & $1 / N$ \\
Grain diameter & $L$ & $1 / N$ \\
Mass & $M$ & $1 / N^{3}$ \\
Kinetic energy & $M L^{2} / T^{2}$ & $1 / N^{3}$ \\
Velocity & $L / T$ & 1 \\
Shear strain rate & $1 / T$ & $N$ \\
Stress & $M /\left(T^{2} L\right)$ & 1 \\
Force (impact load) & $M L / T^{2}$ & $1 / N^{2}$ \\
Bending moment & $M L^{2} / T^{2}$ & $1 / N^{3}$ \\
Inertial time & $T$ & $1 / N$ \\
Flexible barrier stiffness & $M / T^{2}$ & $1 / N$ \\
\hline
\end{tabular}

Table 2. Properties of rigid and flexible barrier (all dimensions in prototype)

\begin{tabular}{lccc}
\hline & Barrier & Stiffness & Inflection point (kN) \\
\hline Rigid & & $\begin{array}{c}\mathrm{EI}=1.88 \mathrm{E} 8 \mathrm{Nm}^{2} \\
\text { at unit width }\end{array}$ & \\
\hline \multirow{3}{*}{ Flexible } & Top cable & $\begin{array}{l}K_{1}=2.6 \mathrm{E} 6 \mathrm{~N} / \mathrm{m} \\
K_{2}=2.0 \mathrm{E} 5 \mathrm{~N} / \mathrm{m}\end{array}$ & 40 \\
& & & \\
& & & 4 \\
& Upper, Lower intermediate, & $K_{1}=1.8 \mathrm{E} 6 \mathrm{~N} / \mathrm{m}$ & 40 \\
& and Bottom cables & $K_{2}=2.0 \mathrm{E} 5 \mathrm{~N} / \mathrm{m}$ & \\
\hline
\end{tabular}


Table 3. Test programme at $22.4 \mathrm{~g}$ and test materials (all dimensions in prototype)

\begin{tabular}{|c|c|c|c|}
\hline $\begin{array}{l}\text { Test } \\
\text { ID }\end{array}$ & Description & Material & $\begin{array}{c}\text { Froude } \\
\text { number } F r\end{array}$ \\
\hline $\mathrm{CS}$ & $\begin{array}{l}\text { Calibration of Sand free flow } \\
\text { Frontal velocity: } 13.3 \mathrm{~m} / \mathrm{s} \\
\text { Flow depth: } 1.01 \mathrm{~m}\end{array}$ & & \\
\hline $\mathrm{RS}$ & Rigid barrier + Sand & $\begin{array}{c}\text { Dry sand } \\
(\mathrm{LB} \text { sand Fraction C) } \\
1530 \mathrm{~kg} / \mathrm{m}^{3}, \varphi=31^{\circ}\end{array}$ & 4.4 \\
\hline FS & Flexible barrier + Sand & & \\
\hline $\mathrm{CL}$ & $\begin{array}{c}\text { Calibration of viscous Liquid free } \\
\text { flow } \\
\text { Frontal velocity: } 12.3 \mathrm{~m} / \mathrm{s} \\
\text { Flow depth: } 1.05 \mathrm{~m}\end{array}$ & Viscous fluid & \\
\hline RL & Rigid barrier + viscous Liquid & $\begin{array}{c}\left(\mathrm{ZnCl}_{2}+\text { Glycerol }+\mathrm{CMC}+\text { water }\right) \\
1580 \mathrm{~kg} / \mathrm{m}^{3}, \eta=11.3 \mathrm{Pas}\end{array}$ & 4.0 \\
\hline FL & Flexible barrier + viscous Liquid & & \\
\hline
\end{tabular}


Table 4. Comparison of peak and static loads (all dimensions in prototype)

\begin{tabular}{|c|c|c|c|}
\hline Test ID & $\begin{array}{c}\text { Normalized } \\
\text { impact height }\end{array}$ & Resultant force $(\mathrm{kN})$ & $\begin{array}{c}\text { Earth pressure } \\
\text { coefficient } \boldsymbol{k}\end{array}$ \\
\hline \multirow{2}{*}{$\mathrm{RS}$} & \multirow{2}{*}{0.55} & 507 (peak) & - \\
\hline & & 424 (static) & $2.3\left(k_{\mathrm{a}}=1.7\right)$ \\
\hline \multirow{2}{*}{ FS } & \multirow{2}{*}{0.54} & 286 (peak) & - \\
\hline & & 260 (static) & $1.5\left(k_{\mathrm{a}}=1.7\right)$ \\
\hline \multirow{2}{*}{$\mathrm{RL}$} & \multirow{2}{*}{1.00} & 884 (peak) & - \\
\hline & & 530 (static) & $1.1\left(k^{*}=1.1\right)$ \\
\hline \multirow{2}{*}{ FL } & \multirow{2}{*}{1.00} & 704 (peak) & - \\
\hline & & 520 (static) & $1.1(k=1.1)$ \\
\hline
\end{tabular}

*The theoretical pressure coefficient for liquid higher than 1.0 is caused by the inclined retaining structure.

Table 5. Comparison of frontal $F r$ and $\alpha$ (all dimensions in prototype)

\begin{tabular}{ccccc}
\hline Test ID & $\begin{array}{c}\text { Velocity } \\
(\mathbf{m} / \mathbf{s})\end{array}$ & $\begin{array}{c}\text { Flow depth } \\
(\mathbf{m})\end{array}$ & $\begin{array}{c}\text { Froude number } \\
\boldsymbol{F r}\end{array}$ & $\begin{array}{c}\text { Dynamic pressure } \\
\text { coefficient } \boldsymbol{\alpha}\end{array}$ \\
\hline RS & $9.0-13.3$ & $\sim 0.5$ & $4.1-5.9$ & $0.4-1.2$ \\
FS & $9.0-13.3$ & $\sim 0.5$ & $4.1-5.9$ & $0.4-0.8$ \\
RL & $10.0-12.3$ & $\sim 1.0$ & $3.2-3.9$ & $1.4-1.6$ \\
FL & $10.0-12.3$ & & & $1.2-1.4$ \\
\hline
\end{tabular}


Figures

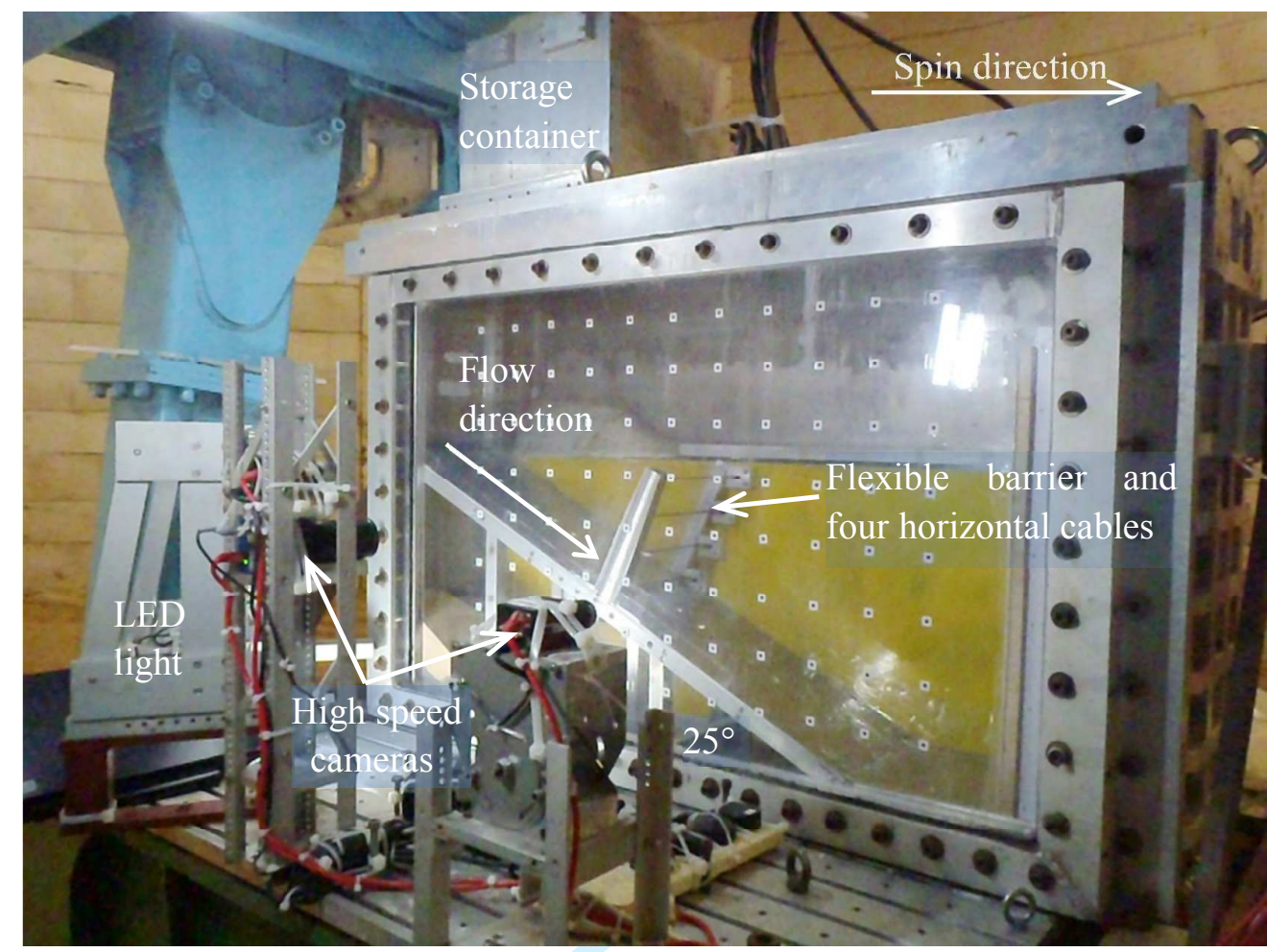

Fig. 1 Side view of model setup and instrumentation 


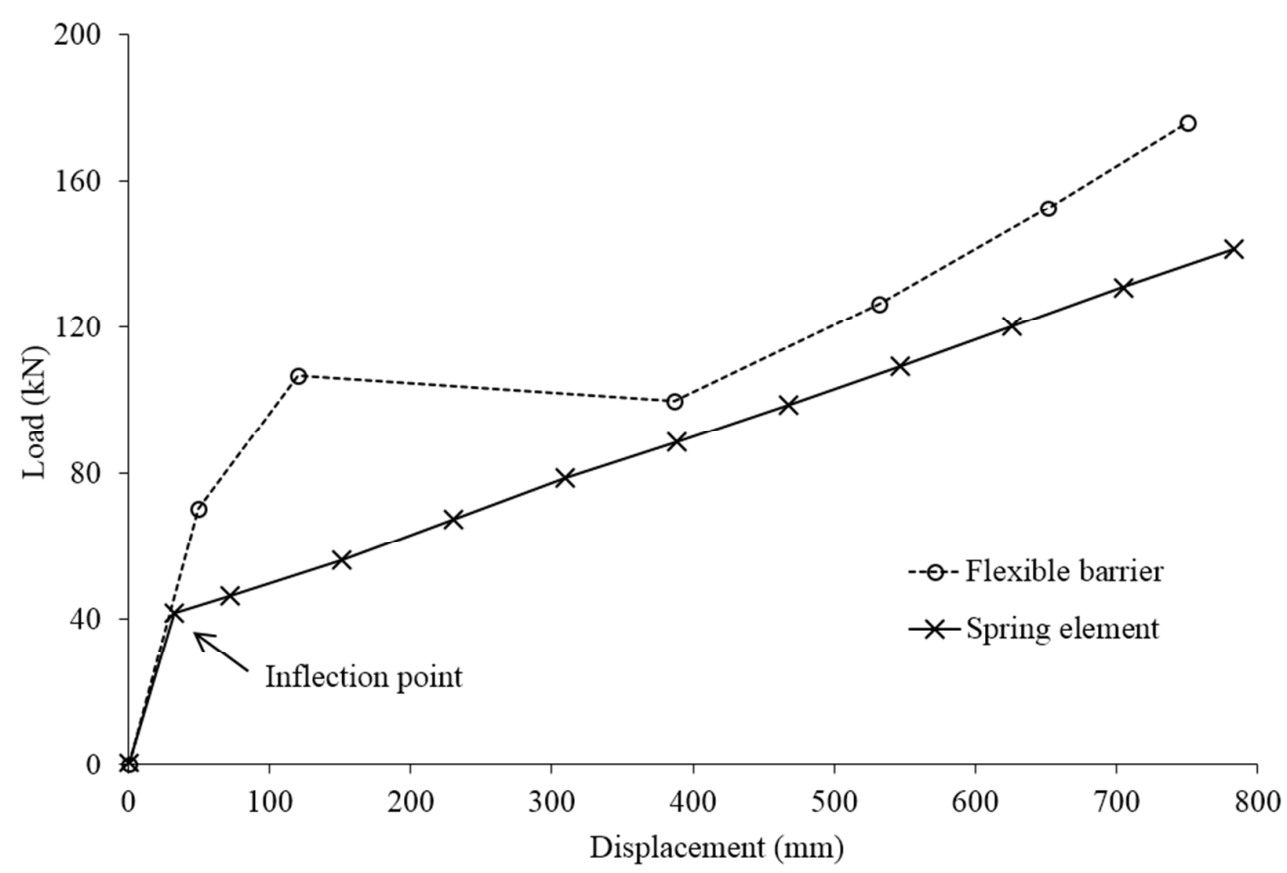

Fig. 2 Load-displacement behaviour of flexible barrier bottom cable (all dimensions in prototype) (Ng et al. 2016) 


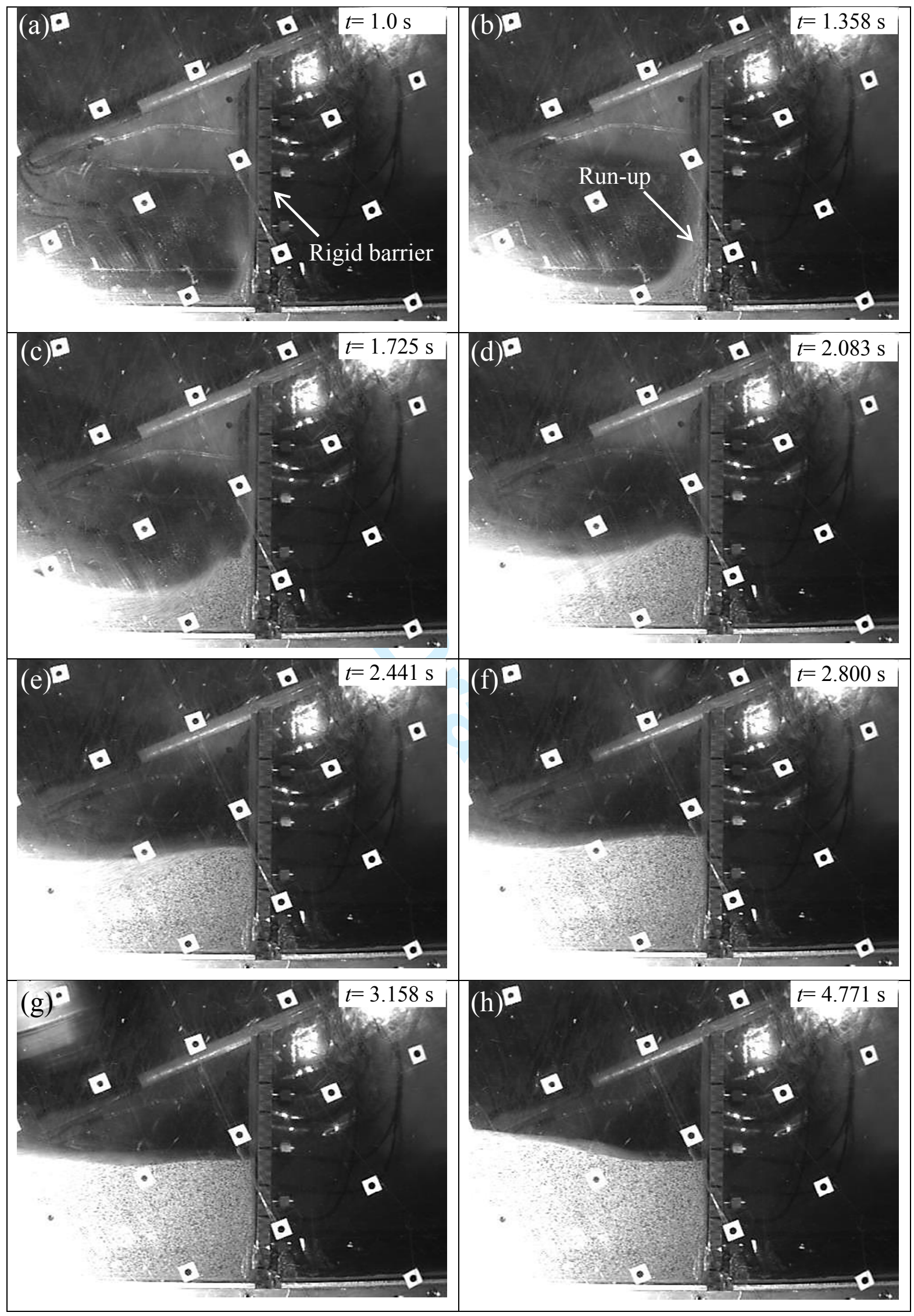

Fig. 3 Observed interaction kinematics for test RS in prototype time: (a) $t=1.0 \mathrm{~s}$; (b) $t=1.358 \mathrm{~s}$; (c) $t=$ $1.725 \mathrm{~s}$; (d) $t=2.083 \mathrm{~s}$; (e) $t=2.441 \mathrm{~s}$; (f) $t=2.800 \mathrm{~s}$; (g) $t=3.158 \mathrm{~s}$; (h) $t=4.771 \mathrm{~s}$. The camera field of view was adjusted flush with the slope $\left(25^{\circ}\right)$. 


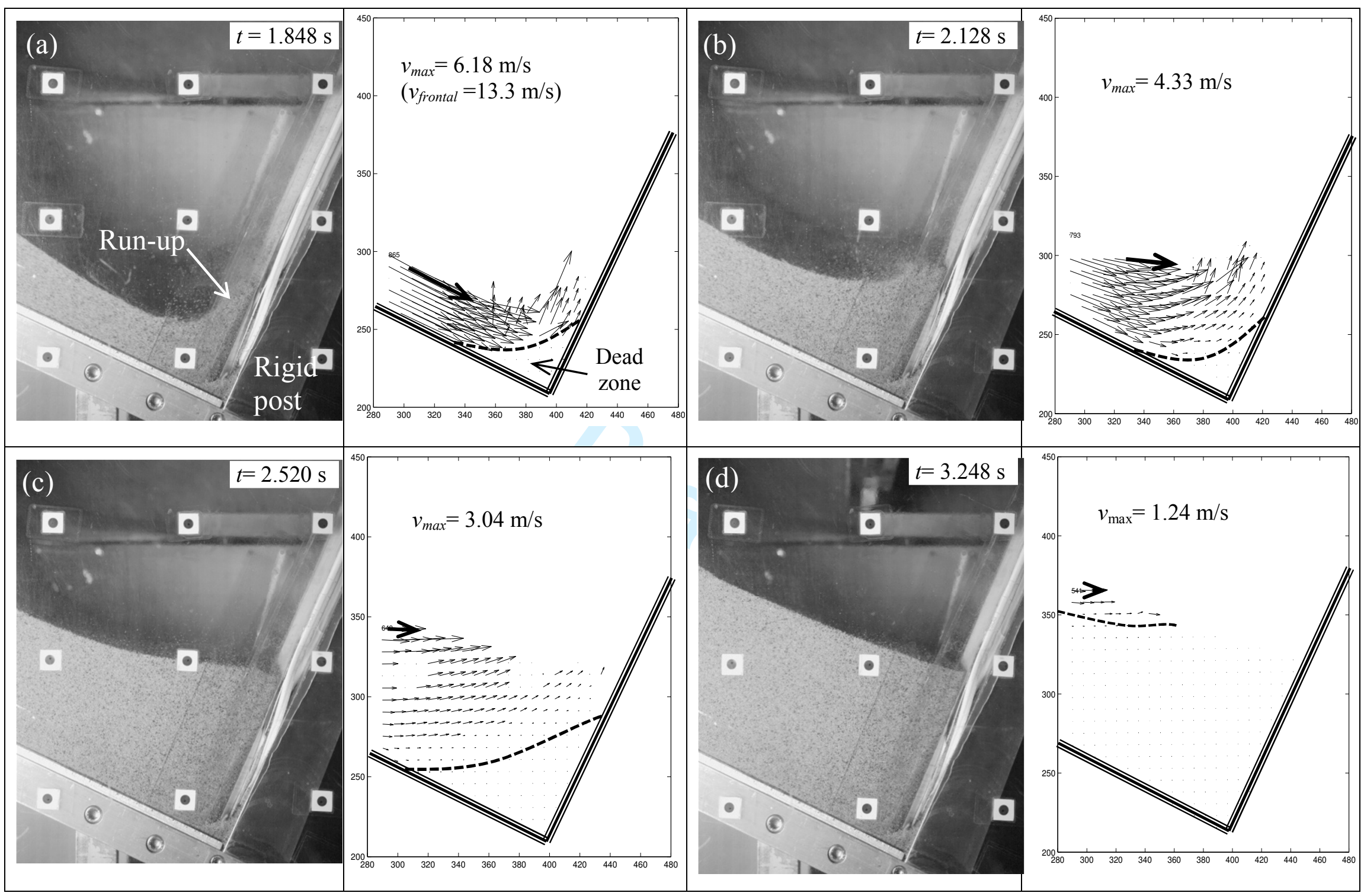

Fig. 4 Observed interaction kinematics for test FS in prototype time: (a) $t=1.848 \mathrm{~s}$; (b) $t=2.128 \mathrm{~s}$; (c) $t=2.520 \mathrm{~s}$; (d) $t=3.248 \mathrm{~s}$. The bold arrows denote the maximum velocity in each figure. 


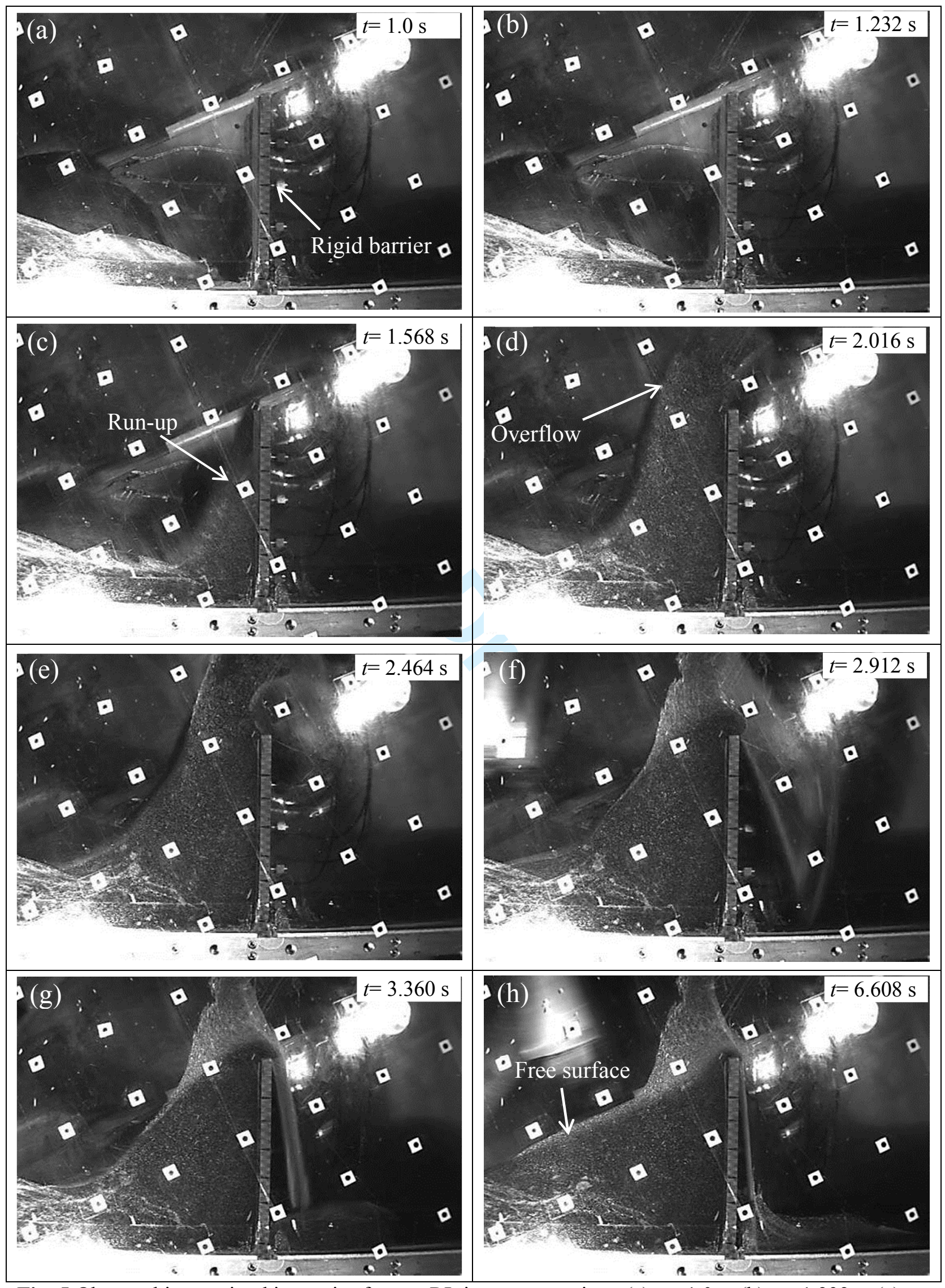

Fig. 5 Observed interaction kinematics for test RL in prototype time: (a) $t=1.0 \mathrm{~s}$; (b) $t=1.232 \mathrm{~s}$; (c) $t=$ $1.568 \mathrm{~s}$; (d) $t=2.016 \mathrm{~s}$; (e) $t=2.464 \mathrm{~s}$; (f) $t=2.912 \mathrm{~s}$; (g) $t=3.360 \mathrm{~s}$; (h) $t=6.608 \mathrm{~s}$. The camera field of view was adjusted flush with the slope $\left(25^{\circ}\right)$. 

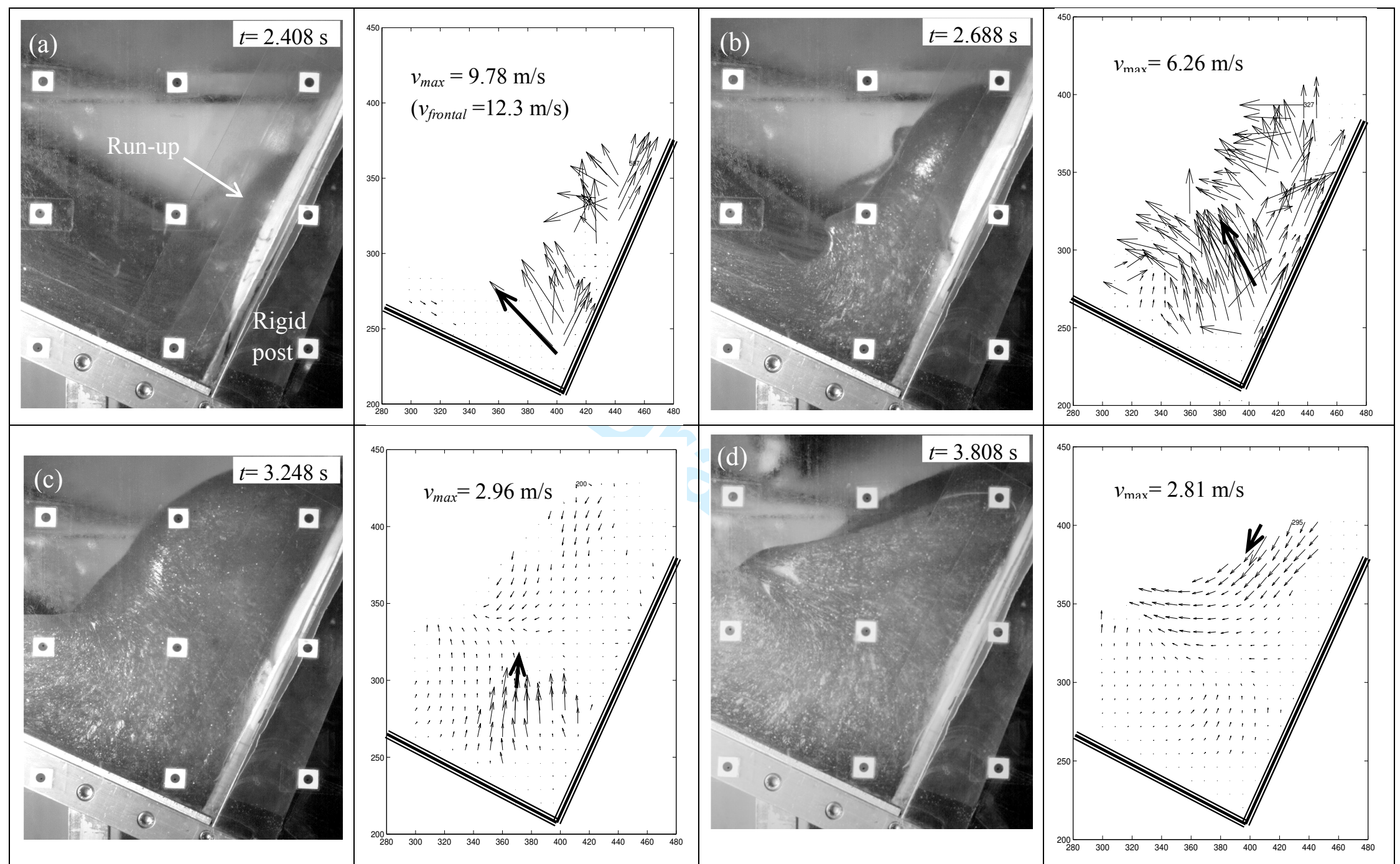

Fig. 6 Observed interaction kinematics for test FL in prototype time: (a) $t=2.408 \mathrm{~s}$; (b) $t=2.688 \mathrm{~s}$; (c) $t=3.248 \mathrm{~s}$; (d) $t=3.808 \mathrm{~s}$. The bold arrows denote the maximum velocity in each figure. 


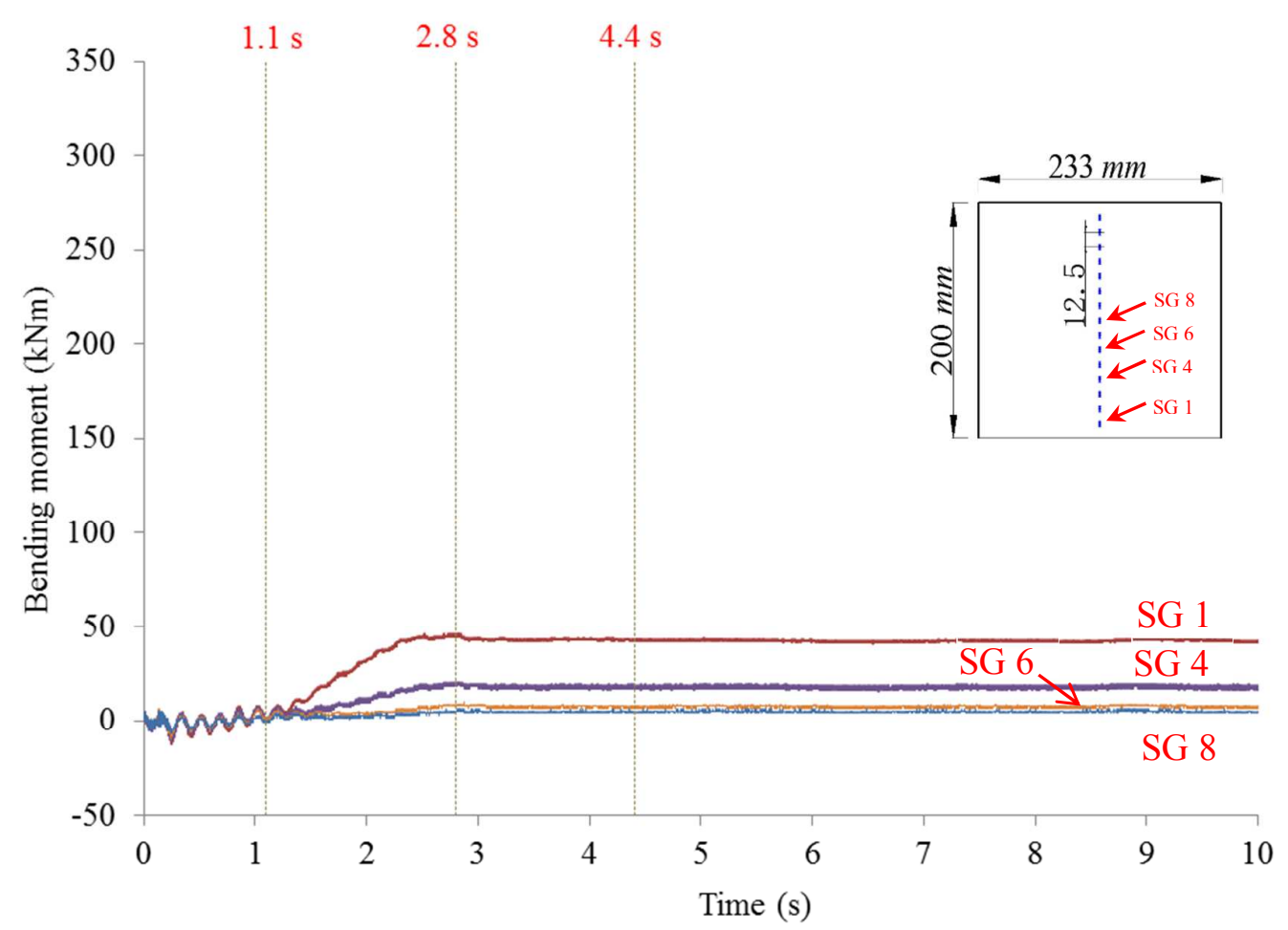

(a)

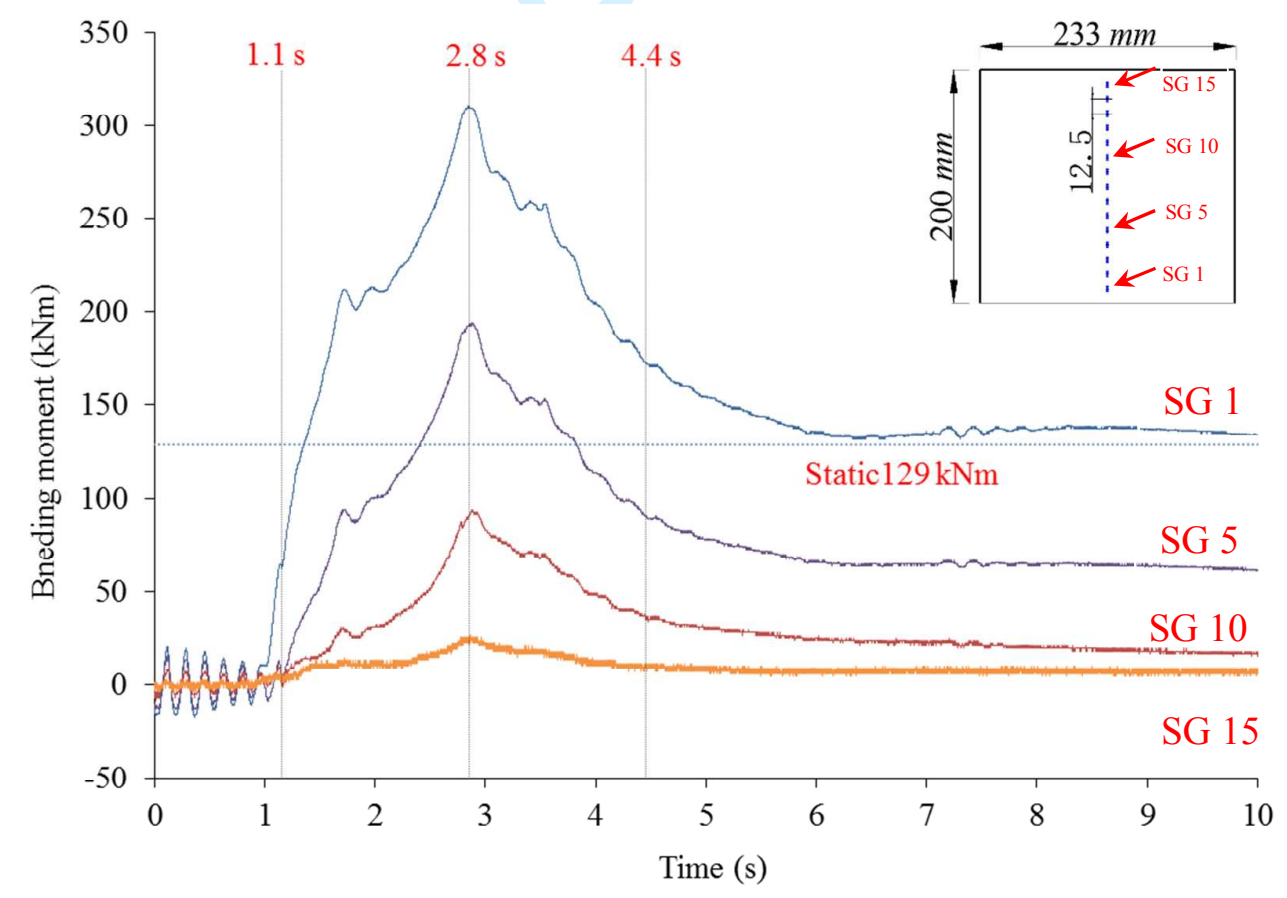

(b)

Fig. 7 Bending moment time history of (a) test RS and (b) test RL (unit width) 


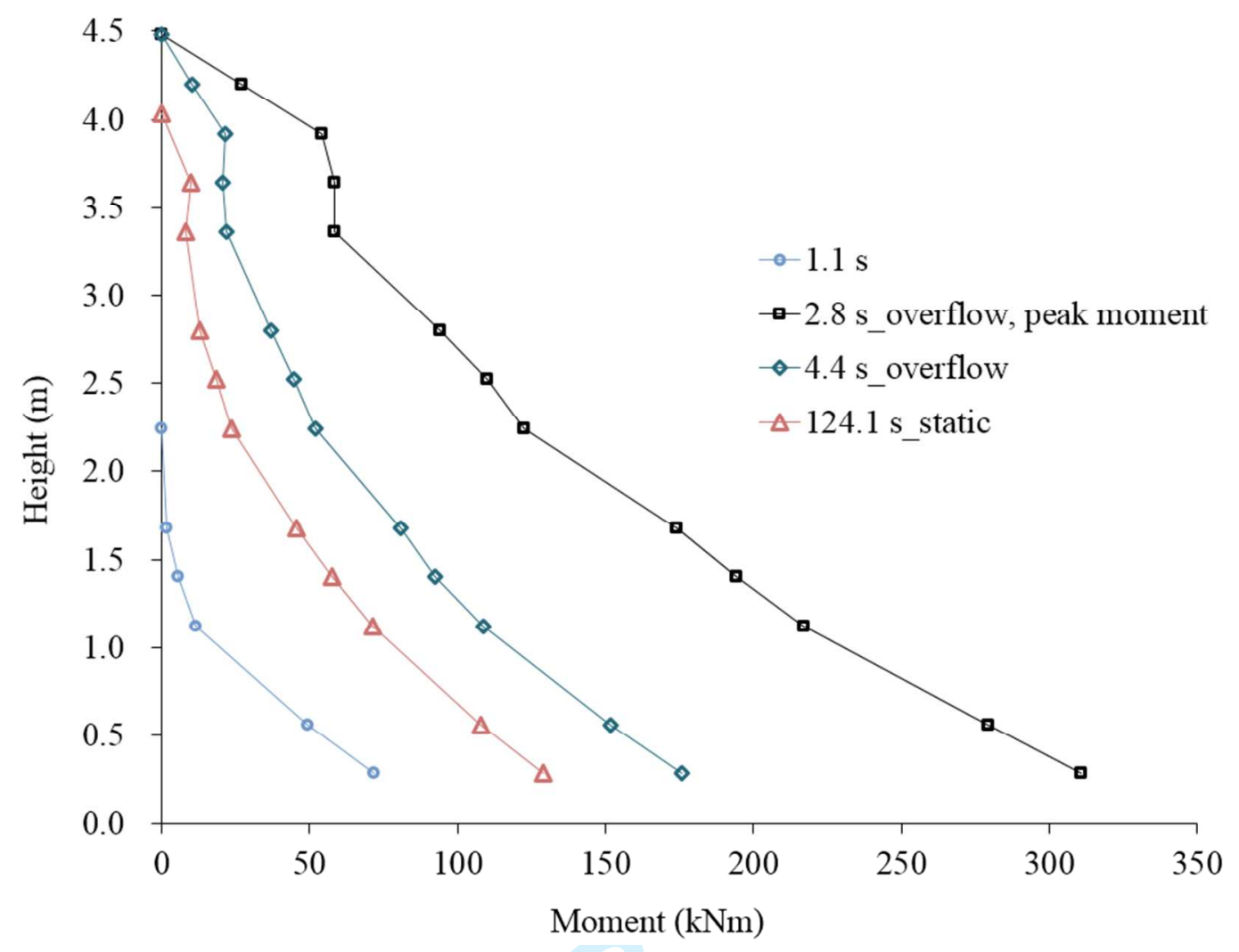

Fig. 8 Bending moment evolution for test RL (unit width) 


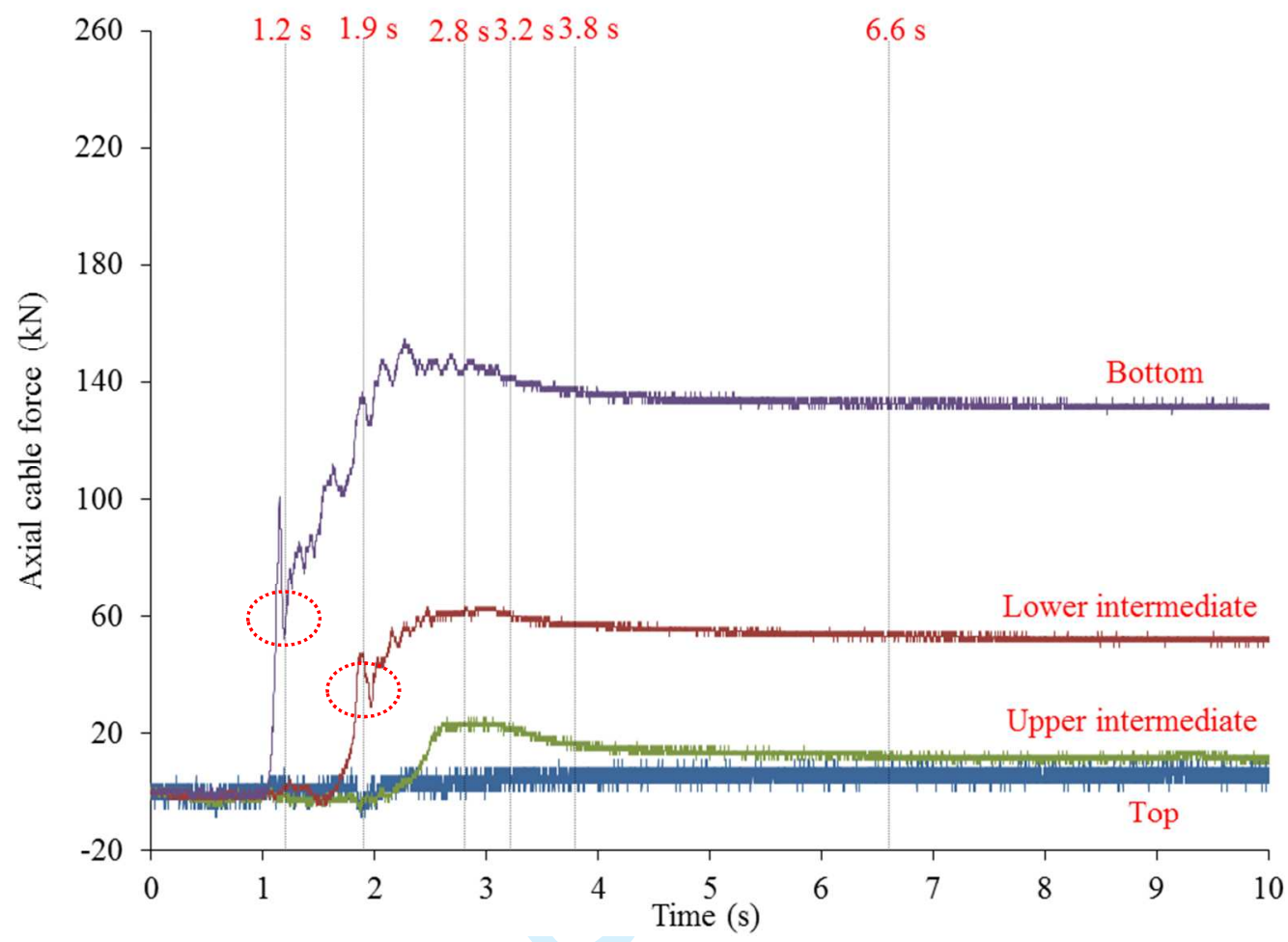

(a)

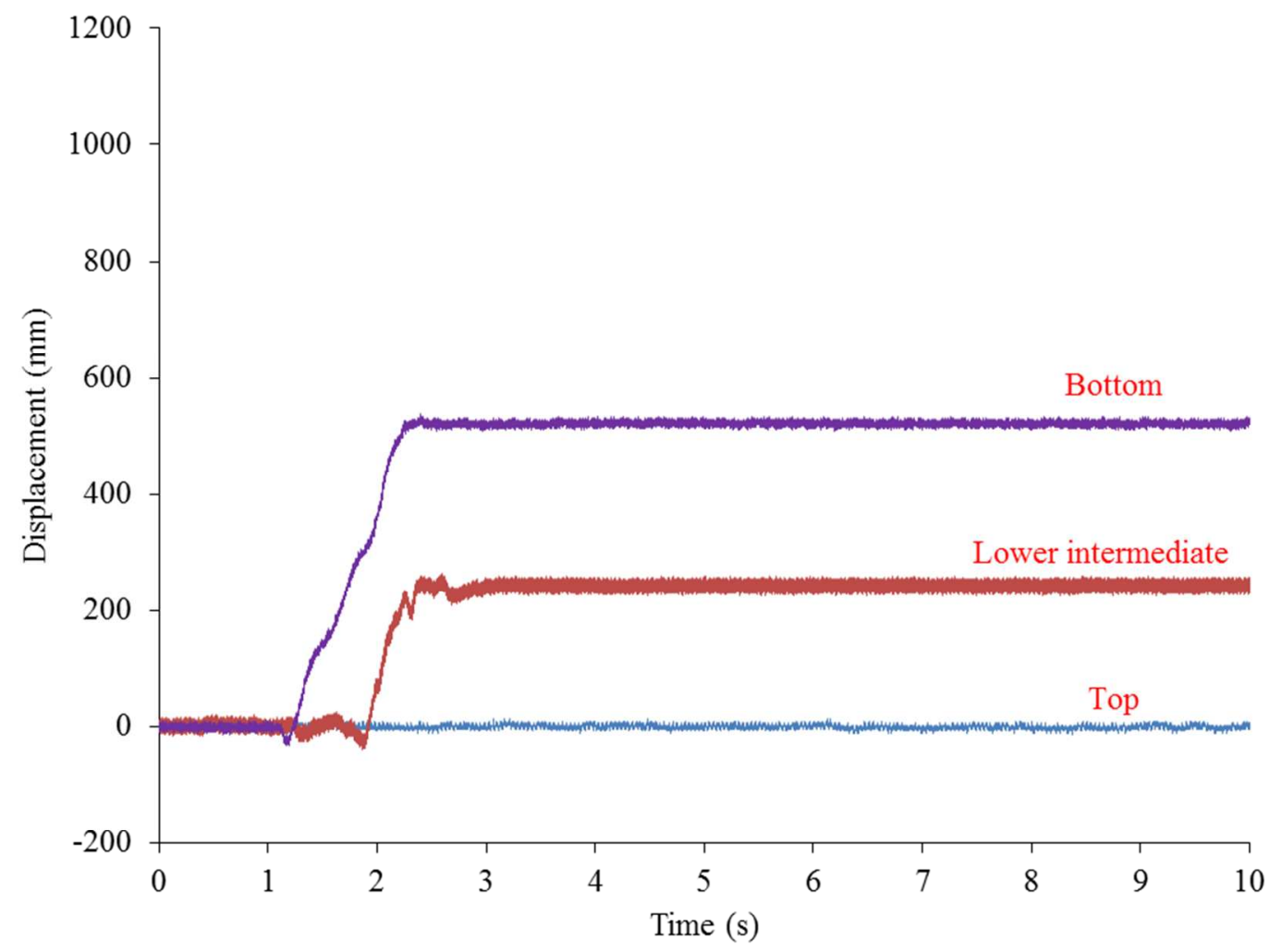

(b)

Fig. 9 (a) cable force time history for test FS (b) cable displacement time history for test FS 


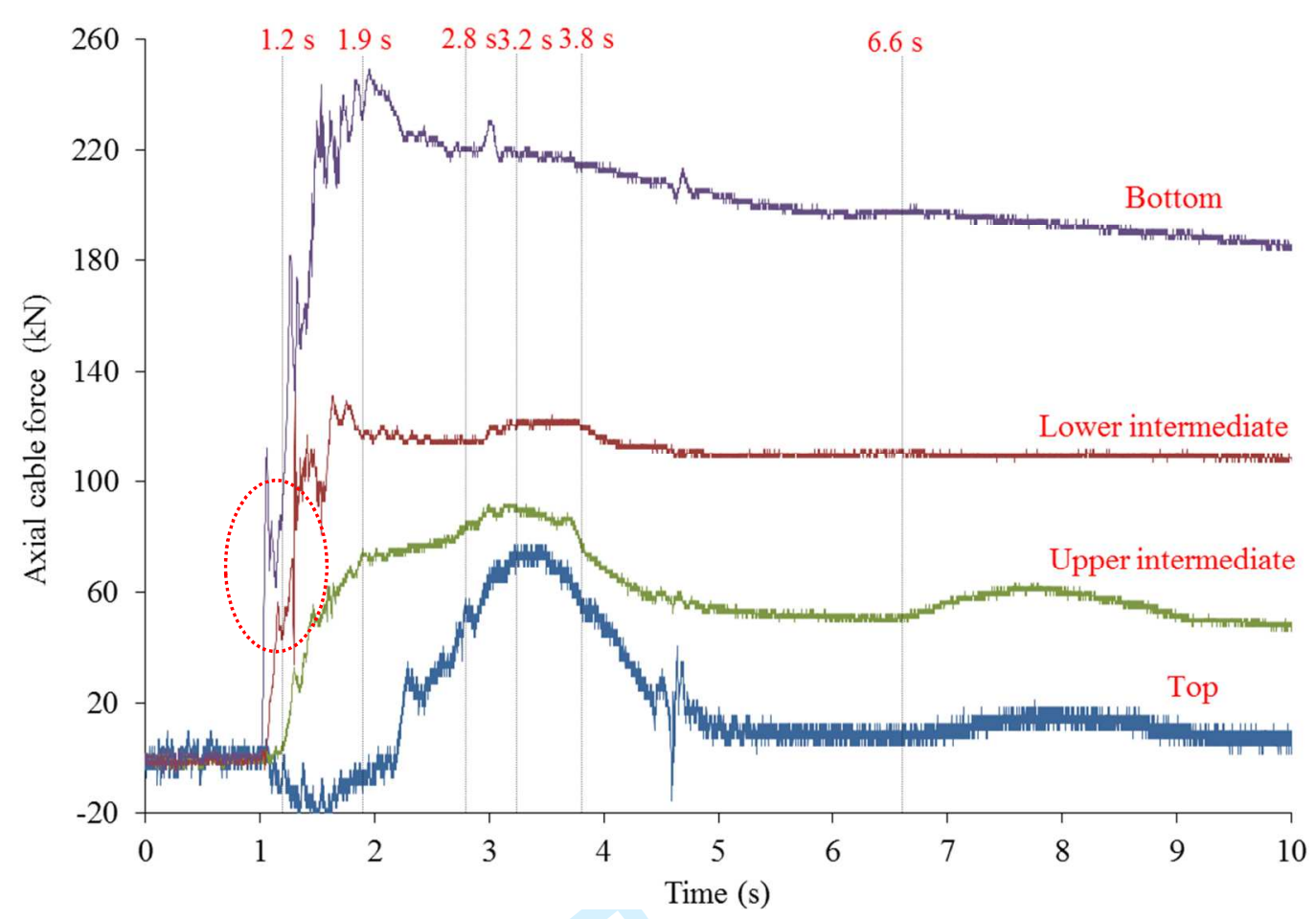

(a)

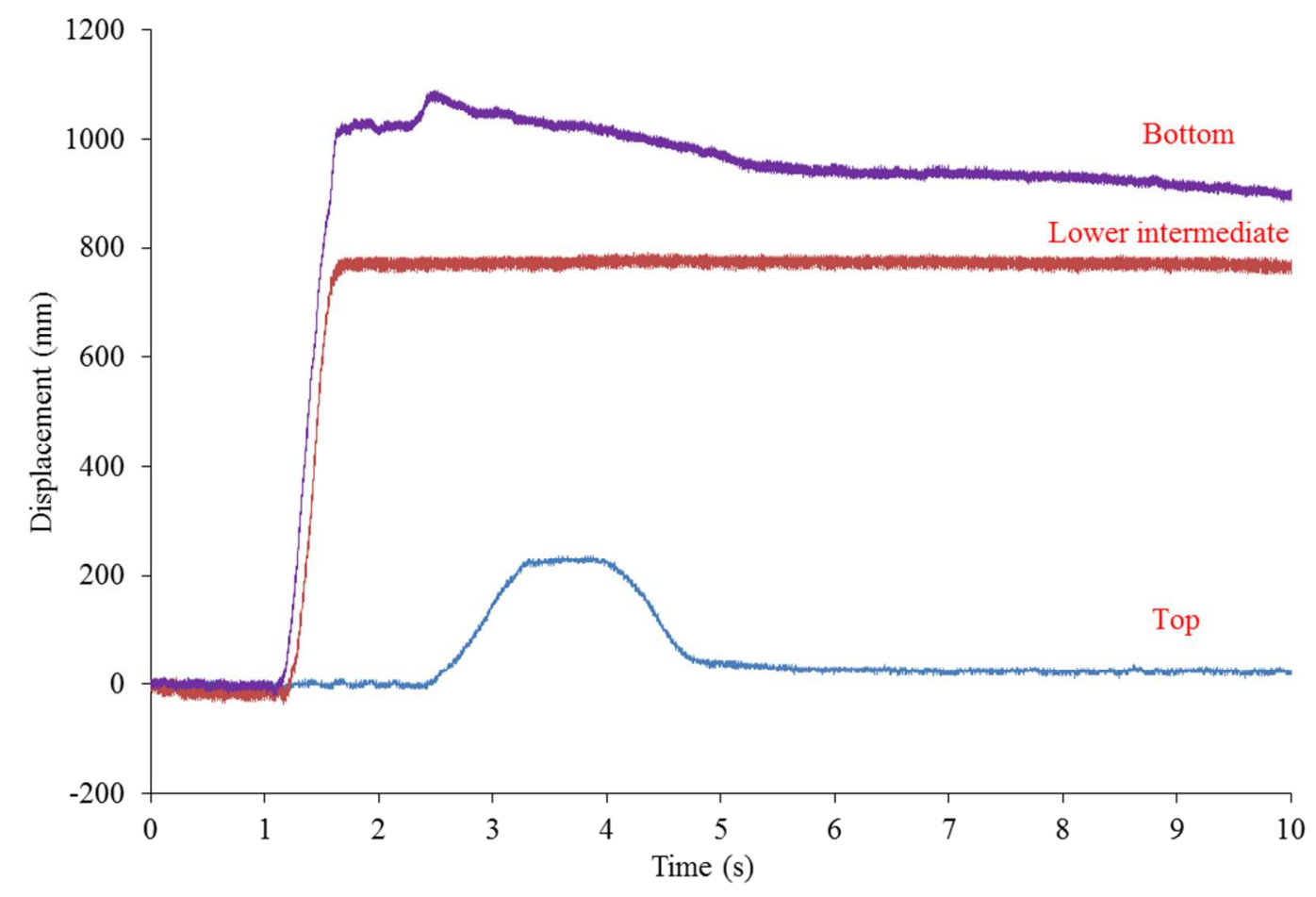

(b)

Fig. 10 (a) cable force time history for test FL (b) cable displacement time history for test FL 


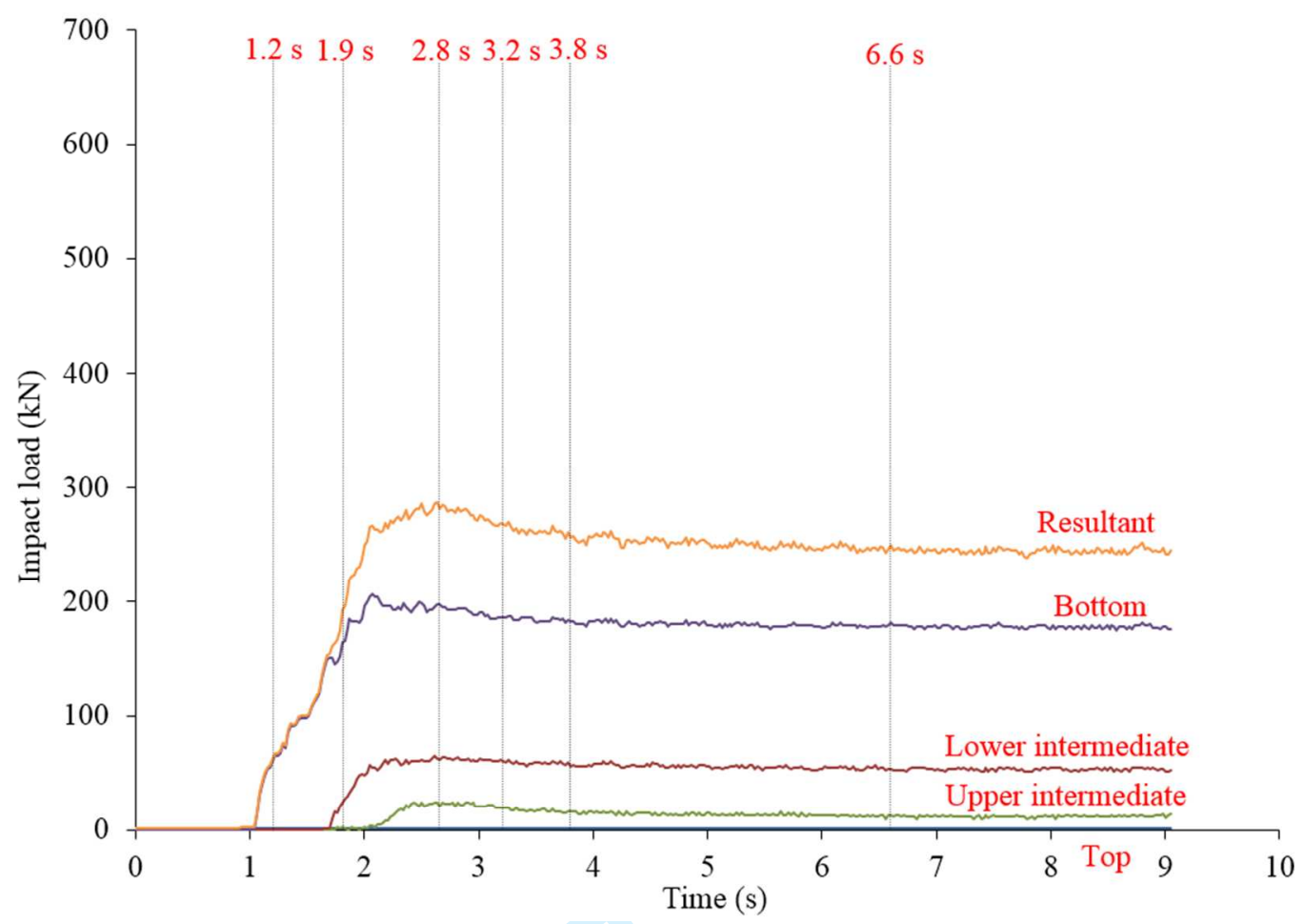

(a)

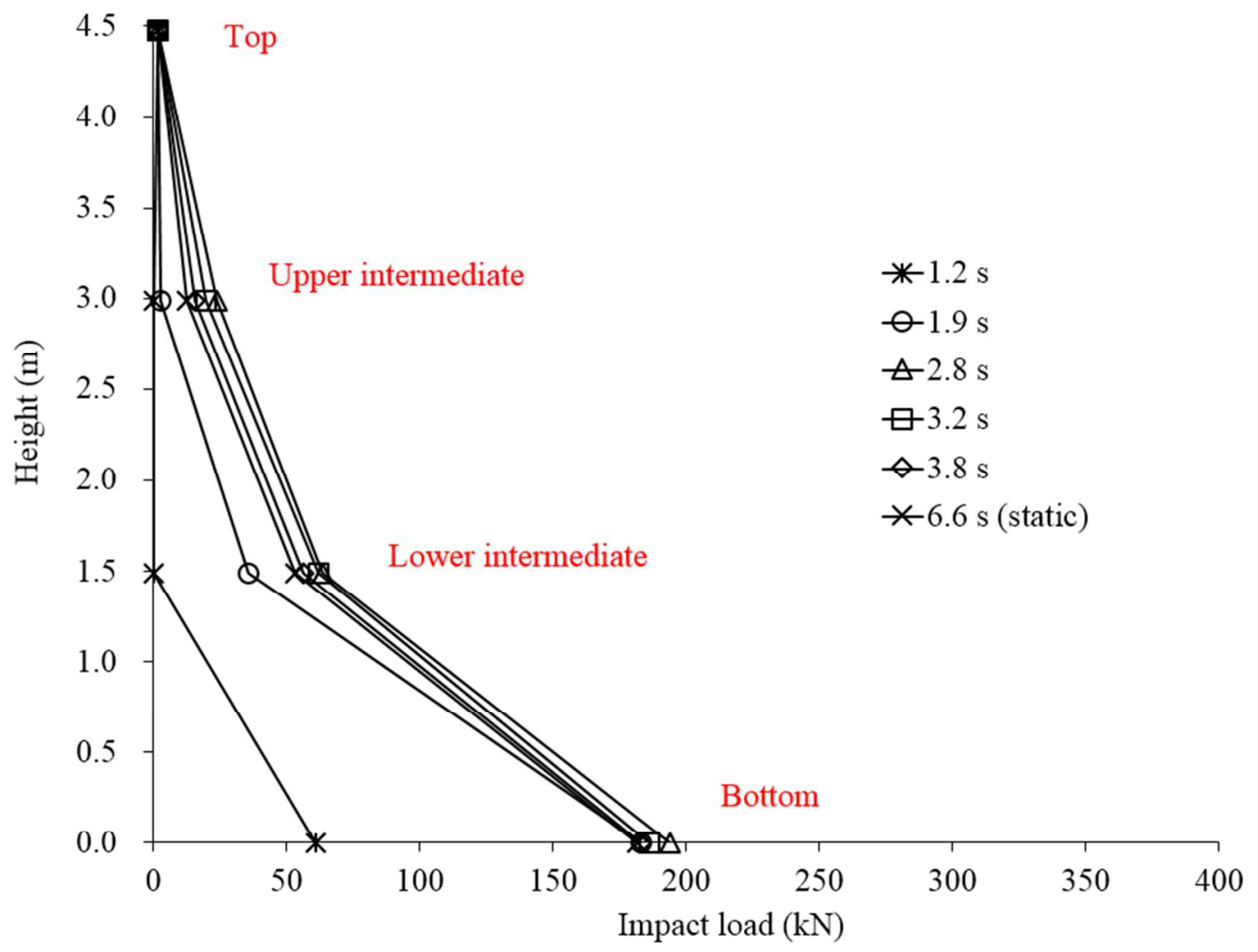

(b)

Fig. 11 (a) resolved impact load time history and (b) impact load evolution for test FS 


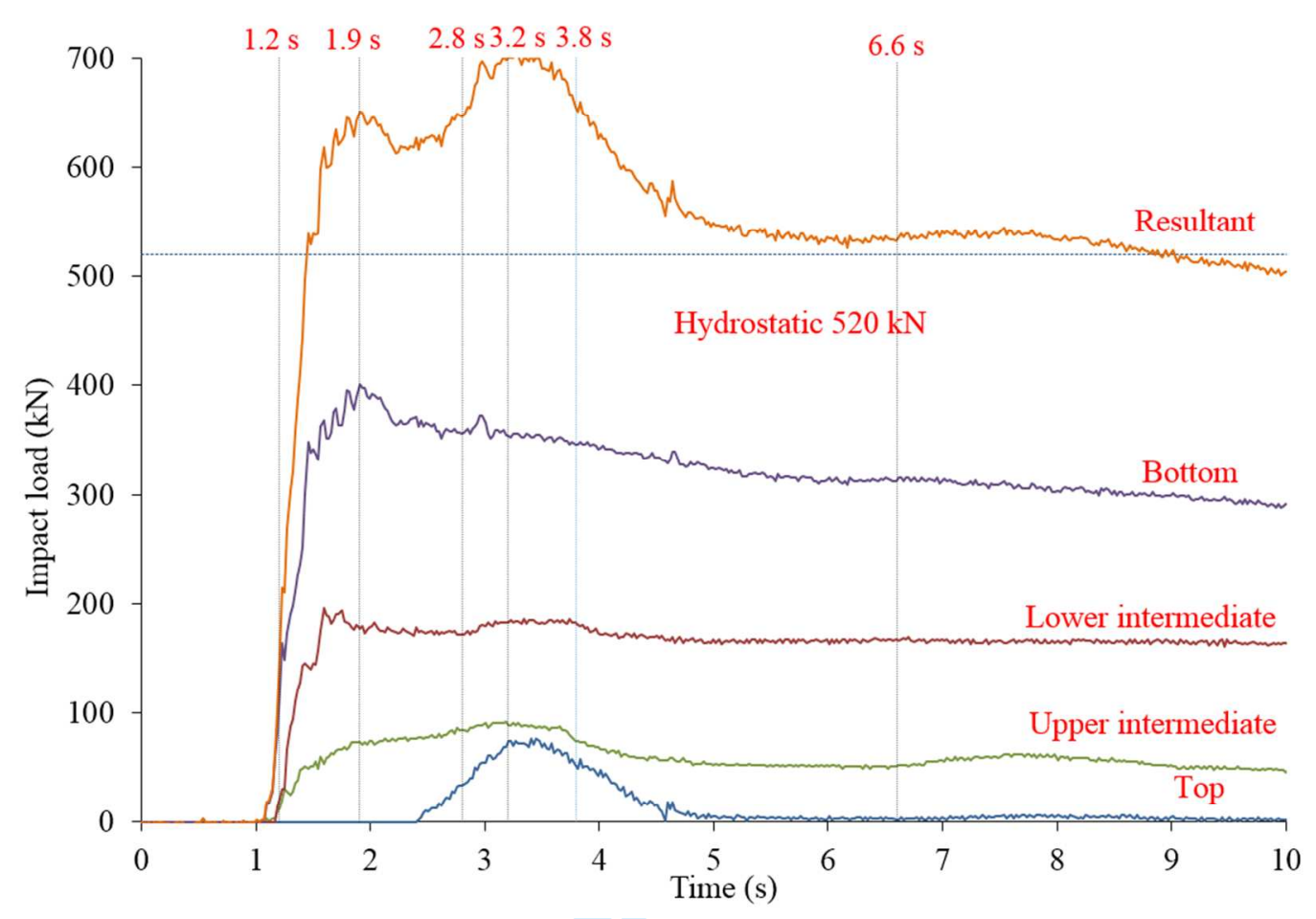

(a)

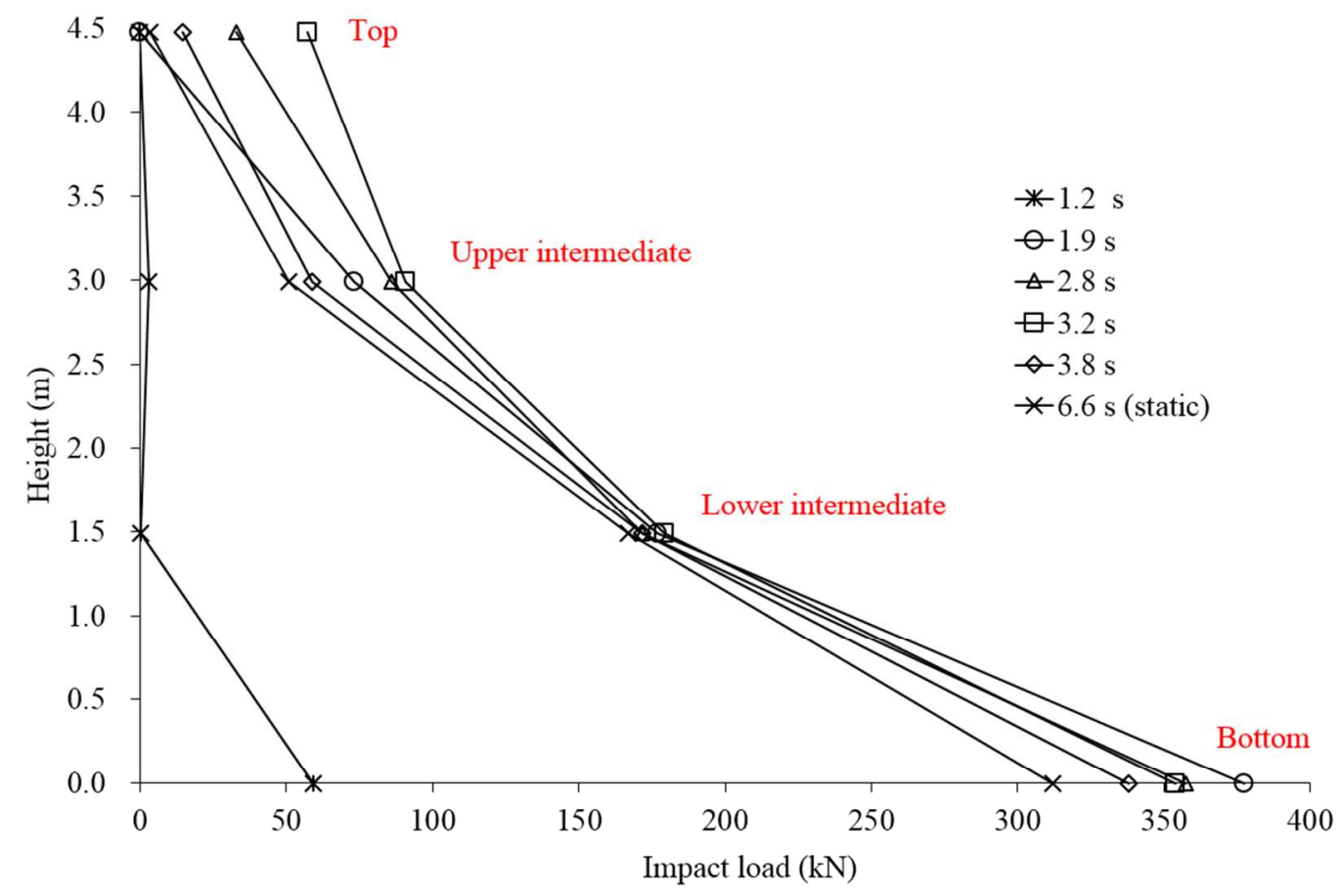

(b)

Fig. 12 (a) resolved impact load time history and (b) impact load evolution for test FL 


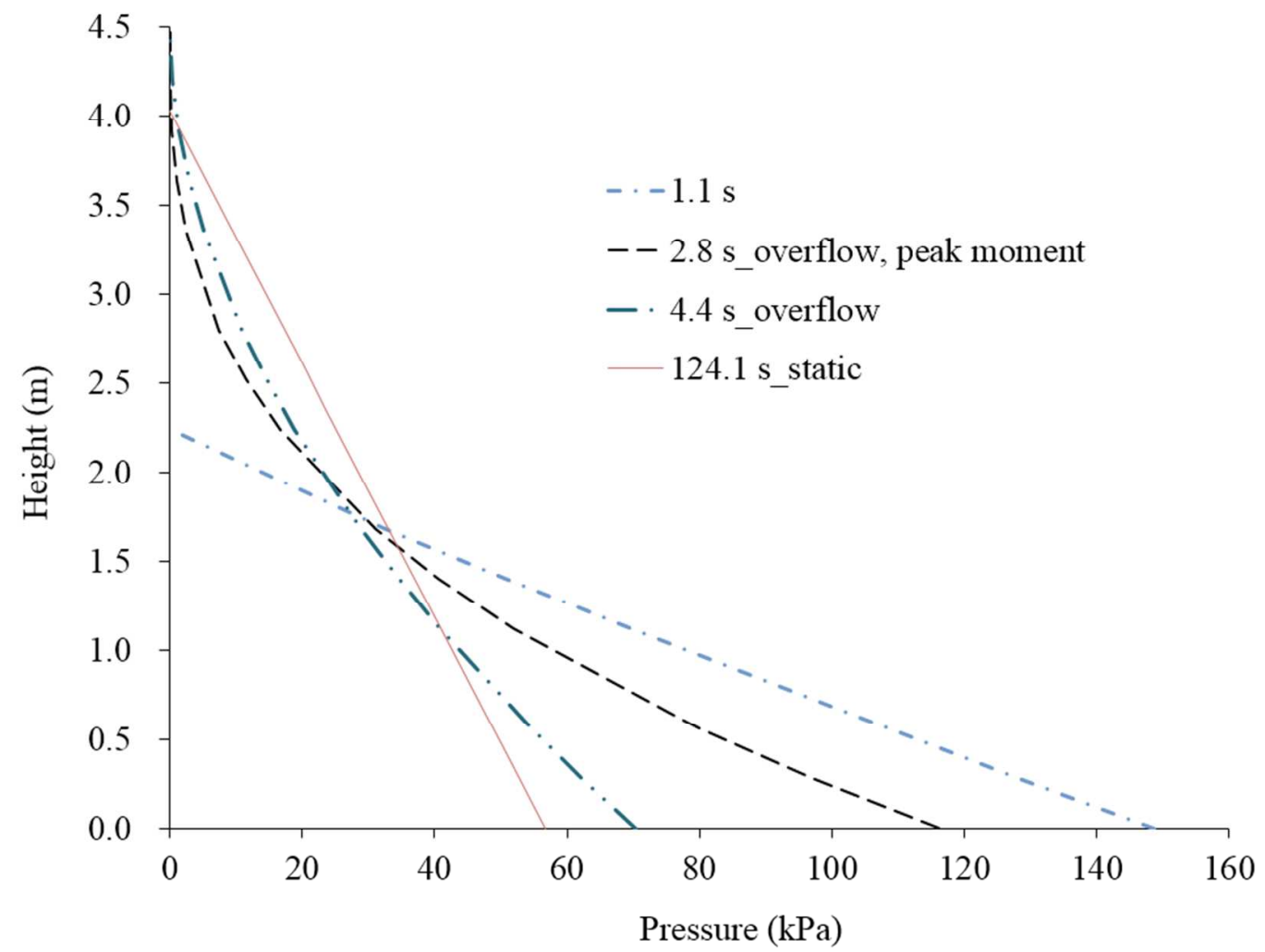

Fig. 13 Impact pressure evolution for test RL 


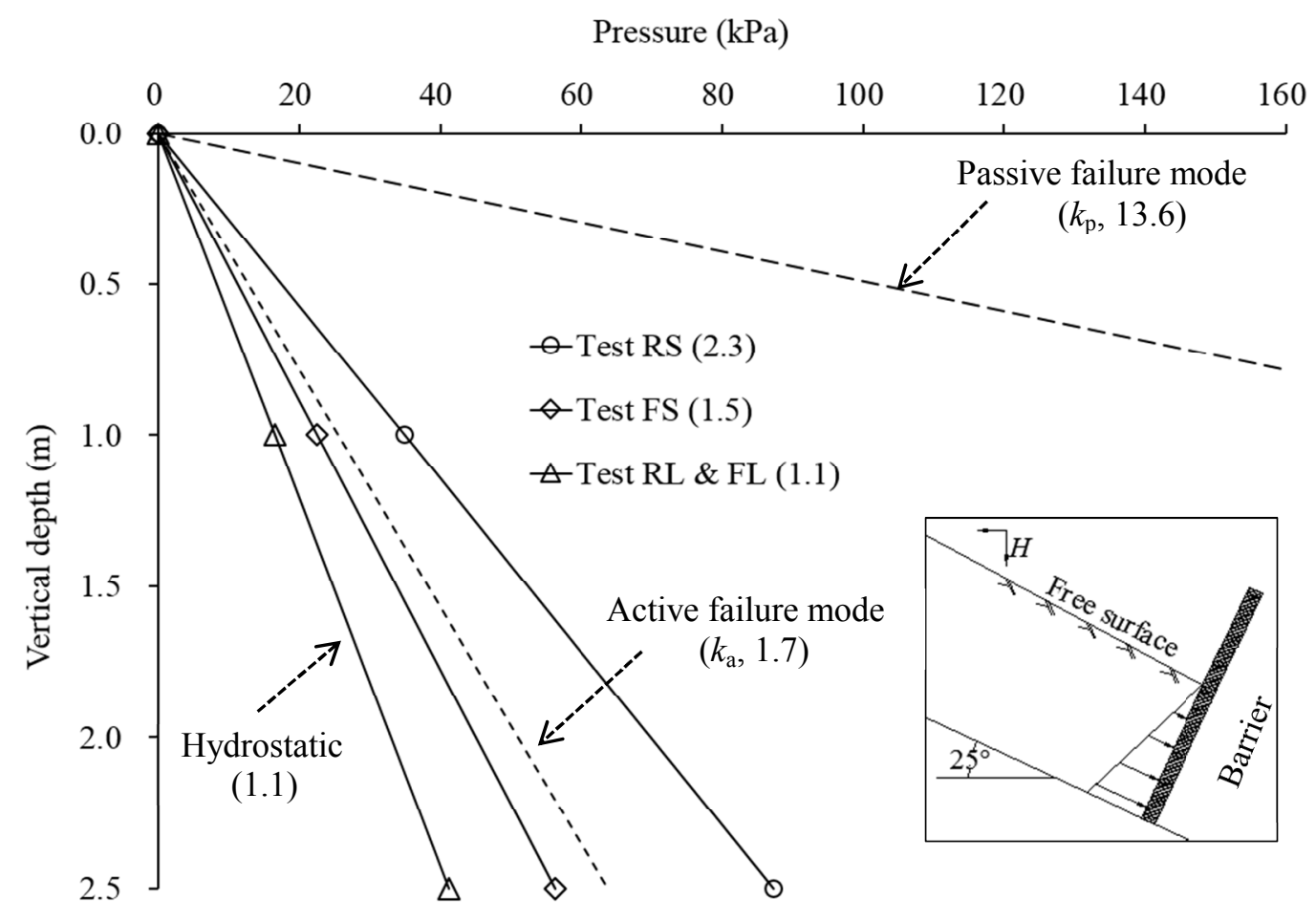

Fig. 14 Static pressure acting on rigid and flexible structures induced by dry sand and liquid. The barrier is $25^{\circ}$ inclined from vertical direction. 


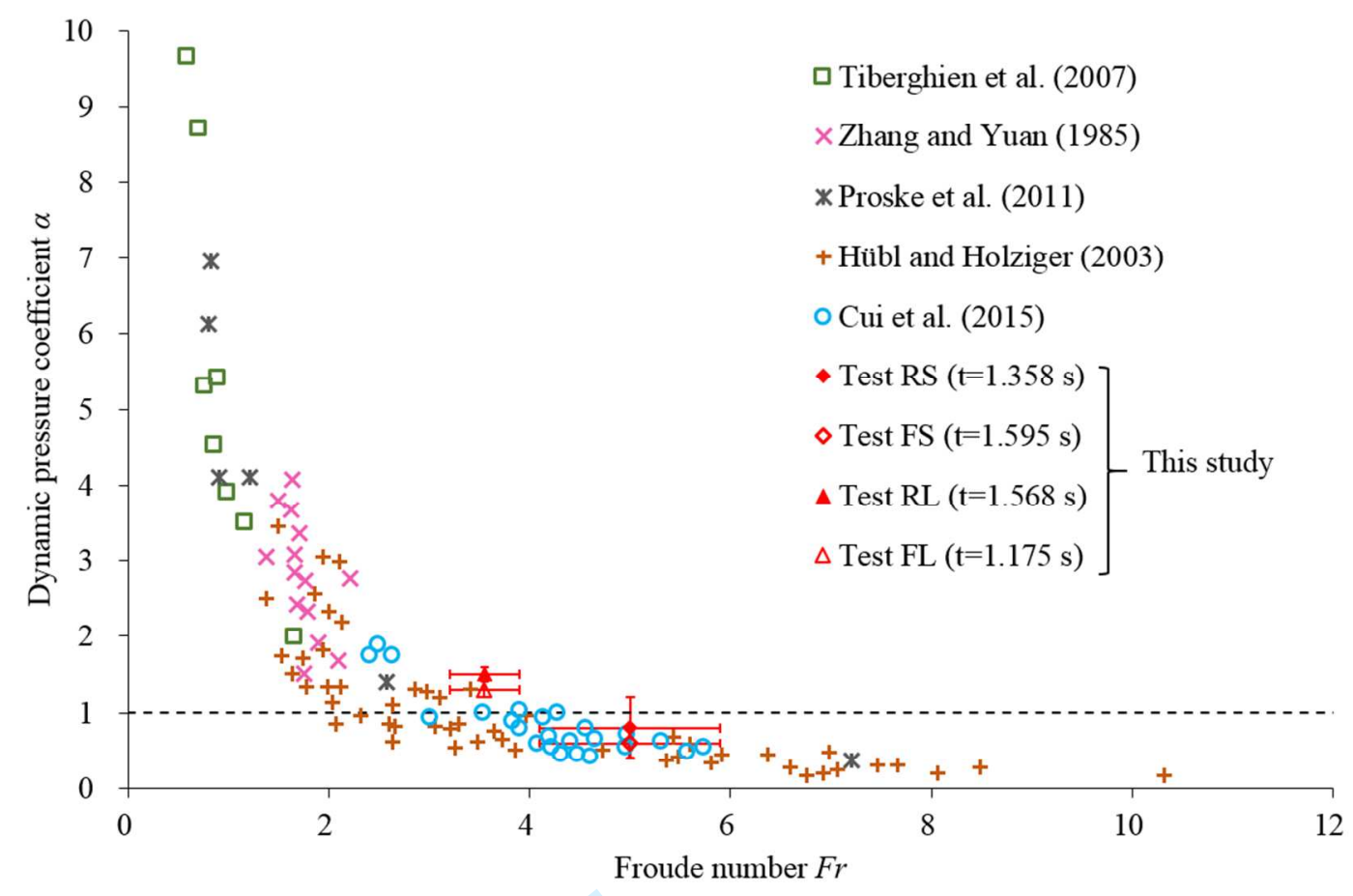

Fig. 15 Relationship between $\alpha$ and $F r$. Except the flexible barrier data points, all the others are from rigid structure tests. 


\section{Appendix. Derivation of cable impact load and cable deflection}

One of the features of the flexible barrier cable is that each cable is positioned horizontally and subject to a relative uniform line load during impact. Due to the large stiffness, the elastic elongation of the cable is estimated only $9 \mathrm{~mm}$ in prototype under the maximum impact load. The loading direction changes slightly when the barrier is deflected. If the direction change is neglected, the loading scenario is analogous with a cable under its own weight when hung only at both ends (Fig. A1).

Mathematically a sagging cable (catenary line) can be described by using a hyperbolic cosine function:

$$
y=a \cosh \frac{x}{a}
$$

where $a$ is the only parameter of this function.

The following time-histories of test FS and FL are available: barrier width $L$, arc length $S(=L+$ cable elongation), and cable force $T$.

i. Determination of $a$. The arc length of a catenary line between two coordinates $\mathrm{P}_{1}\left(x_{1}, y_{1}\right)$ and $\mathrm{P}_{2}\left(x_{2}\right.$, $\left.y_{2}\right)\left(x_{2}>x_{1}\right)($ Fig. A1)

$$
S=a \sinh \frac{x_{2}}{a}-a \sinh \frac{x_{1}}{a}
$$

Let $x_{2}=L / 2, x_{1}=-L / 2$, the arc length of a whole catenary line can be used to determine the parameter $a$ as follows

$$
S=2 a \sinh \frac{L / 2}{a}
$$

ii. $\quad$ Slope of the curve at L/2 (Fig. A2).

$$
\tan \psi=\left.y^{\prime}\right|_{L / 2}=\sinh \frac{L / 2}{a}
$$

iii. The total impact force

$$
P=2 V=2 T \sin \psi=2 T \frac{\tan \psi}{\sqrt{1+\tan ^{2} \psi}}=2 T \tanh \frac{L / 2}{a}
$$


iv. The deflection of the cable. The vertical difference of two coordinates $\mathrm{P}_{1}\left(x_{1}, y_{1}\right)$ and $\mathrm{P}_{2}\left(x_{2}, y_{2}\right)\left(x_{2}>\right.$ $\left.x_{1}\right)$ (Fig. A2).

$D=a \cosh \frac{x 2}{a}-a \cosh \frac{x 1}{a}$

Let $x_{2}=L / 2, x_{1}=0$, the deflection of a catenary line can be determined as follows

$D=a \cosh \frac{L / 2}{a}-a$ 


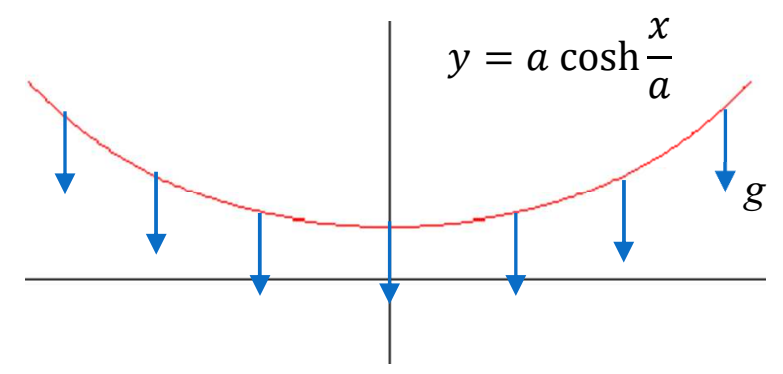

Fig. A1. A catenary line

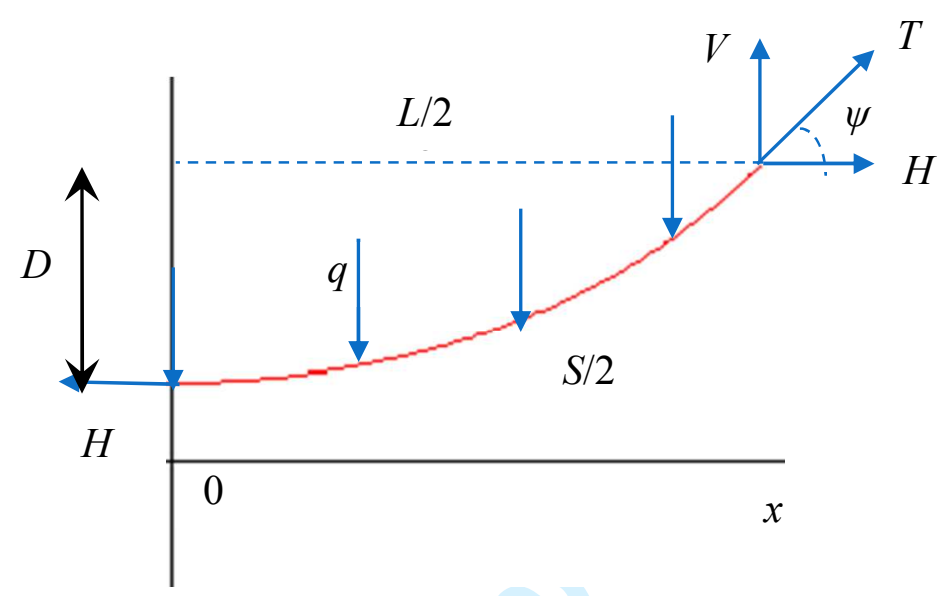

Fig. A2. Decomposition of the force within a catenary line at the hanging point 